\title{
A CONTINUOUS BI-LEVEL MODEL FOR THE EXPANSION OF HIGHWAY NETWORKS February 15, 2013
}

\author{
Eusebio Angulo ${ }^{1}$, Enrique Castillo ${ }^{2}$ \\ Ricardo García-Ródenas ${ }^{1}$, and Jesús Sánchez-Vizcaíno ${ }^{3}$ \\ ${ }^{1}$ Department of Mathematics, \\ University of Castilla-La Mancha, Spain \\ ${ }^{2}$ Department of Applied Mathematics and Computational Sciences, \\ University of Cantabria, Spain \\ ${ }^{3}$ Department of Civil Engineering, \\ University of Castilla-La Mancha, Spain
}

\begin{abstract}
Adding new corridors to a highway network represents a multicriteria decision process in which a variety of social, environmental and economic factors must be evaluated and weighted for a large number of corridor alternatives. This paper proposes a new bi-level continuous location model for expansion of a highway network by adding several highway corridors within a geographical region. The upper level problem determines the location of the highway corridors, taking into account the budgetary and technological restrictions, while the lower level problem models the users' behavior in the located transport network (choices of route and transport system). The proposed model takes into account the demand in the area served by the new network highway corridors, the available budget and the user behaviour. This model uses geographical information in order to estimate the length-dependent costs (such as pavement and construction costs) and the cost of earth movement. The proposed method is tested using the Standard Particle Swarm Optimization algorithm and applied to the Castilla-La Mancha geographic database. The previous methodology has been extended to a multiobjective approach in order to handling uncertainty in demand.
\end{abstract}

Keywords: Highway corridors location - Highway networks - Demand covering Heuristics.

\section{Introduction}

Addition of new highways to an existing road network is a complex problem that involves many factors (construction, environmental and user costs, political issues, etc). It belongs to the field of network expansion problems and investment decision-making, one of the suggested topics of applications for this special issue of Computers and Operation Research. Traffic network management problems involve optimal decisions about the improvement of a transportation system considering user's behaviour. Notable examples 
of this type of problem are: Continuous Network Design Problem (CNDP), Toll Pricing Problem, Signal Setting Problem, OD matrix Adjustment Problem (DAP) etc.

The framework for the formulation of this type of problems is bi-level programming. The so-called lower-level problem is defined by a traffic assignment model and includes user behaviour (the followers in a Stackelberg game) in a traffic network while the upperlevel problem models the decisions of the transport planner (the leader in a Staclkelberg game). This is a hierarchical decision process which cannot be modelled by a bi-objective approach in which the objectives of each follower and of the leader are placed in the same level.

Traffic network design problems can be divided into two categories:

i) Enhancement capacity of sub/urban road networks. This problem is known as the continuous network design problem (CNDP) [55]. CNDP assumes that the topology of the network is fixed and that the intention is to establish an optimal parametrization of the network for the existing demand. CNDP considers urban networks in which congestion is present and that travel times are flow-dependent in modelling mathematically the effect of congestion in the network.

ii) Design of the layout of suburban traffic networks, such as rural highway networks. This problem deals with the building of new roads to improve existing road networks. In this type of problem the topology of the network is modified and it is assumed that congestion is negligible.

[54] and [21] propose also two bi-level programming models that solve with Particle Swarm Optimization but for congested urban networks.

In this paper the problem of adding new highway corridors to an existing rural highway network in a given geographical context is addressed. We have reviewed a large collection of papers in the existing literature and the most common hypothesis is to consider that congestion is negligible for this type of situation. (see references [26], [28], [30], [33], [34]). A review of this topic is the work in [35].

Initially, we can classify the different approaches to solve the problem in two main groups: the Discrete Network Design Problem (DNDP) and the Highway Alignment Optimization (HAO) problem (see [38]).

The DNDP consists of a macroscopic highway design, but the number of possible solutions in space is finite. A good example, can be seen in [14] who proposed a discrete location approach to solve a wider range of problems. This model has been widely applied to transportation, logistic, telecommunications and production-distribution systems. The interested reader can consult the surveys of [44], [47] and [2]. These problems are formulated as multicommodity network flow problems and can deal with large networks, which are generally modeled as graphs. Some interesting examples of techniques that have been developed to solve the DNDP are the Branch-and-Bound (see [4] and [17]), the Modified Quasi-Optimization heuristic (see [52]) and [13]), the Lagrangian Relaxation (see [20]) or 
heuristic algorithms such as Simulated Annealing, Genetic Algorithms, etc (see [15] and [40]).

The HAO is a microscopic highway route design problem and a continuous location model, whose input data are the highway endpoints. The inputs are used to calculate the alignment that minimizes a certain combination of costs and requirements, such as construction costs (right-of-way, pavement and earthwork costs), user costs (travel time, vehicle operating and accident costs), environmental costs (noise, air pollution and wetland loss), design constraints (horizontal alignment, vertical alignment and cross-section) and geographical constraints. The costs and constraints involved in HAO problems are analyzed in detail in [32] and [35]. There are various studies dealing with HAO problems and most of them are solved by means of genetic algorithms and the help of Geographic Information Systems (GIS) ([28]; [27]; [26]; [23]; [24]). Authors have improved sequentially these problems, for example, [29] added to previous work the maintenance costs, which has been traditionally ignored in the past. [41] and [42] developed methods for locally optimizing intersections within HAO problems. [25] considered the traffic demand in the optimization problem. Although these models have acquired a high level of sophistication they all assume that the highway endpoints and the demand are already known. In addition, due to the high computational cost they are unable to optimize large networks.

There are many mathematical models in the existing literature that have been developed for optimizing three-dimensional (3D) alignments (see, for example [8]; [22]; [33]; [24]; [34]; [30]; [7]; [31]; [36]; [35]; [37] and [38]), from which most of them deal with the HAO problem.

Recently, [38] proposed a bi-level method that incorporates to the HAO problems the demand, the route location and all the costs above mentioned. The problem of this work is that it only locates optimally the connection of two highway corridors. [1] proposes a demand-based approach to provide a set of potential corridors. The problem is formulated as a continuous location model which seeks a set of optimal corridors with respect to the demand of potential users while satisfying budget constraints. This work is a microscopic problem that allows locating of a highway corridor without considering the existing network.

In this paper we introduce a model that can be considered as a hybrid of DNDP and HAO. As such, it shares advantages of both methods and allows us to dealt with macroscopic highway design (a global view), considers demand and route choice, and incorporates the different costs with the exception of the three-dimensional (3D) alignments. This should not be a problem, because our model must be considered as a previous tool that supplies input to any of the HAO models described above that consider the 3D alignments. More precisely, our method locates several possible corridors (defined by their endpoints) to enlarge an existing network, taking into account the demand and the costs. This method allows for a great flexibility in the definition of the costs involved in the alignment. The GIS permits to measure specific costs in the study area which represent the environmental and construction costs as a function of the geological characteristics 
of the terrain, the cost of land purchase, earth movement and/or the need to include junctions or bridges. From these estimates the model works with a bivariate linear interpolation function and defines the costs as the curvilinear integral with respect to the interpolated cost function.

Table 1 shows the basic differences between the DNDP, the HAO problem, a bi-level problem solved in [38] and our Continuous Network Design Problem (CNDP*).

Bi-level mathematical programming is difficult to solve owing, fundamentally, to bad mathematical properties and huge size. A sign of this difficulty is the fact that various methods have been developed for the simplest case of the bi-level linear problem (BLP). An excellent review of the exact methods for solving mixed integer bi-level linear problems based on enumeration and reformulation techniques is given in [51].

This has meant that the algorithms applied to real transport problems are heuristic in nature. Very few exact methods for solving the bi-level models can be applied to real large scale transport problems. [50], [56], [43], [18] and [19] propose optimization methods for these bi-level models which have certain properties of local convergence.

Currently, one strategy for the application of exact methods in solving real bi-level problems of large scale is to apply decomposition methods. [51] propose an approach based on Benders decomposition method for mixed integer bi-level linear problems. [18] and [19] apply a simplicial decomposition scheme to DAP.

In the CNDP* model developed, only the lower-level problem is known explicitly. This necessitates the use of heuristic solution methods. Particle Swarm Optimization [39] is a type of random search method applied successfully to a large number of problems. [54] and [21] apply it to the CNDP and show numerically that it has significant convergence properties. This paper adapts the Standard PSO to the model described and develops parallelization techniques to accelerate convergence.

The rest of the paper is organized in the following way: Section 2 sets out in detail the mathematical models constructed in order to approach the problem. Section 3 explains the methods used for solving the models. In Section 4, computational experiments are carried out on a real case study. Finally, the last section of the paper provides some conclusions and future research.

\section{A continuous location model}

Bi-level mathematical programming consists of two nested optimization models, the socalled lower level or follower's problem and the upper level or leader's problem. The upper level variables act as parameters of the objective function and/or the restrictions on the lower level. This is the appropriate framework in this type of transport network design problem. The upper level determines the location of the highway corridors, considering budgetary and technological restrictions, while the lower level models users' behaviour in the located transport network (route and transport system choices, in this model). 


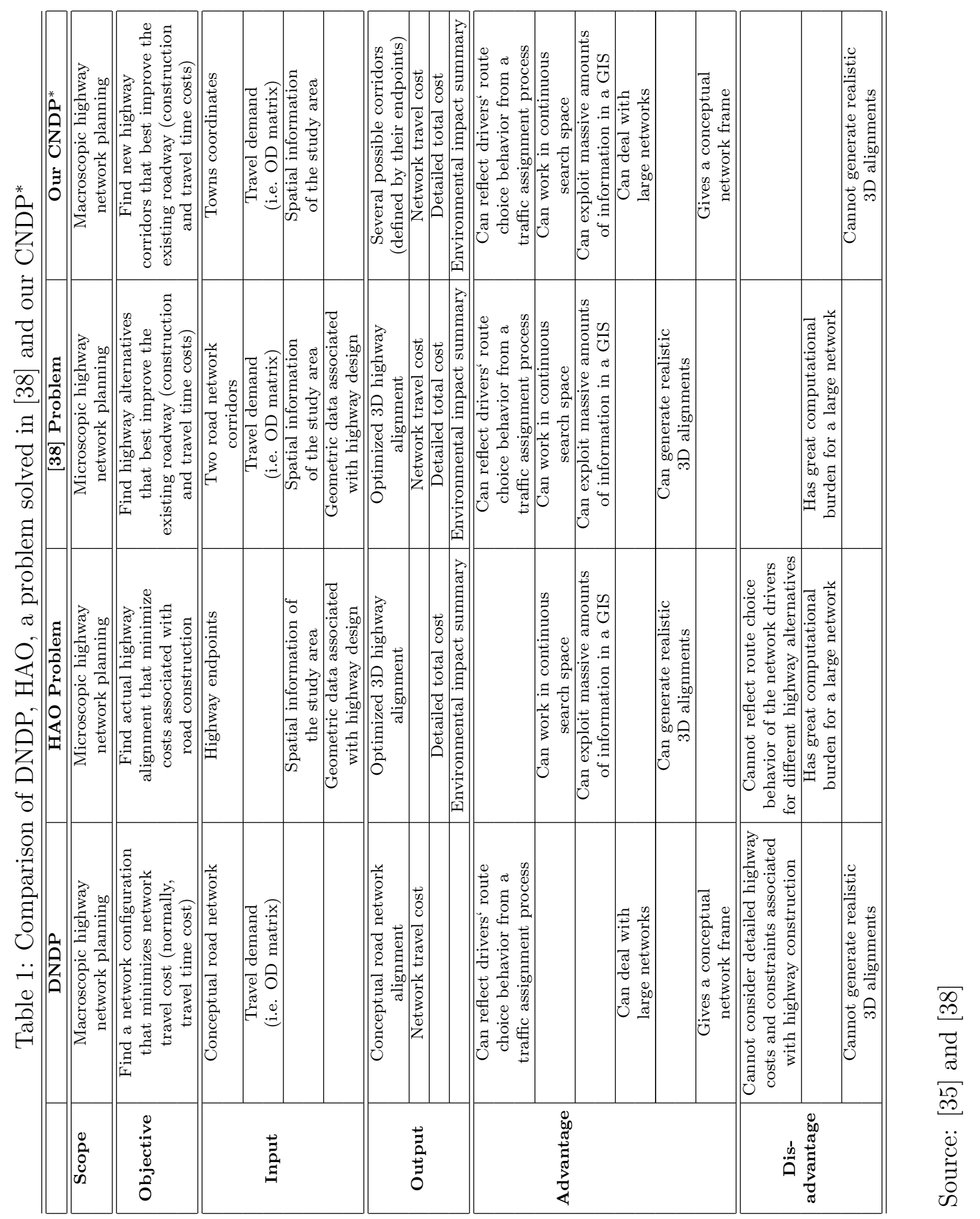


Schematically, the proposed model has the following structure:

\section{$\left[\mathrm{CNDP}^{*}\right]$}

$$
\begin{array}{cc}
\text { Minimize } & Z(u) \\
x, y & \\
\text { subject to: } \quad & K(x, y) \leq B_{0} \\
& u=H(x, y),
\end{array}
$$

where the objective function $Z(u)$ is the total travel time of all users, which depends on the vector $u$ of travel times associates with the ODs, $K(x, y)$ is the construction cost function, which depends on the vectors $x=\left(x_{1}^{-}, x_{1}^{+}, x_{2}^{-}, x_{2}^{+}, \ldots, x_{n}^{-}, x_{n}^{+}\right)$and $y=$ $\left(y_{1}^{-}, y_{1}^{+}, y_{2}^{-}, y_{2}^{+}, \ldots, y_{n}^{-}, y_{n}^{+}\right)$, which contain the two endpoint coordinates of the $n$ highway corridors and define the new highway corridors. Superindices ${ }^{+}$and ${ }^{-}$are used to indicate the direction of the transport facility: flow goes from $\left(x_{i}^{+}, y_{i}^{+}\right)$to $\left(x_{i}^{-}, y_{i}^{-}\right)$, with $i=1, \ldots, \widehat{n}$ (new and existing highway network). $B_{0}$ is the available budget and $H(x, y)$ is the travel time cost function.

The objective function (1) represents the network design criterion, and will be analysed in subsection 2.1. Restriction (2) represents the budgetary limitations and is analyzed in subsection 2.2. Finally, restriction (3) provides $u$, the transport costs (travel time) in the new network, as a function of the routing decisions made by the users of the new infrastructure. This relation is defined implicitly and is the result of applying several optimization methods (see [12]). It corresponds to the lower level of the bi-level scheme, and is described in subsection 2.3.

The resulting optimization model is a complex nonlinear problem.

\section{$2.1 \quad$ Objective function}

The model considers a transport system with three networks:

$\mathcal{N}_{a}$ : The current highway network. It corresponds to the existing main road system.

$\mathcal{N}_{b}$ : The new transport network to be located. It corresponds to the set of new highways.

$\mathcal{N}_{c}$ : The network giving access to $\mathcal{N}_{a} \cup \mathcal{N}_{b}$. It consists of the secondary road network. It is assumed that this network gives access to any point of network $\mathcal{N}_{a} \cup \mathcal{N}_{b}$.

On the other hand, the model considers that there exist places on the plane, called centroids and denoted $c \in \mathcal{O}$, from which the users begin (demand origin) and finish (demand destination) their journeys. It is assumed that the coordinates of these points $\left(x_{c}, y_{c}\right), c \in \mathcal{O}$ are known. 
The distance between a centroid and an arbitrary point $(x, y)$ of a highway corridor is calculated using the Euclidean distance:

$$
d=\sqrt{\left(x-x_{c}\right)^{2}+\left(y-y_{c}\right)^{2}} .
$$

A set of demand pairs $w=(O, D)$ between centroids $O, D \in \mathcal{O}$ is considered. The set of all these pairs is represented by $W$, and $g_{w}$ is the number of users who wish to make a journey of type $w \in W$. The information contained in $\left\{g_{w}\right\}_{w \in W}$ is called the origin-destination demand matrix, which summarizes the mobility pattern of the potential demand of the transport system. It is assumed that the origin-destination matrix is known and fixed during the period under study and that all the travellers use the network of minimum generalized travel time. This assumption does not mean that the demand is held constant over the period of study (several years), but rather that we work with mean values for this period.

The objective function (1) of the optimization model is defined by the design criteria. The transport network design problems can be classified in two groups:

1. Competing networks. In this approach the new facilities compete with the existing transport network. The aim sought is to capture the greatest possible demand, which implies reducing the level of service in the existing network. One example of this group is [45] who uses an optimization criteria for reducing congestion in the traffic network.

2. Complementary networks. This approach considers the new facility as an expansion of the existing network. The aim sought is to optimize the resulting joint network by, for example, minimizing the total transport time in the whole transport system. One example of this methodology is that followed in the advancement of the socalled combined trips such as park'n ride. To solve our problem of adding new corridors to a highway network the most appropiate alternative is this approach, in which the aim is to minimize the total transport time in the system.

Since we consider more appropiate the second group, we minimize the total transport time in $\mathcal{N}_{a} \cup \mathcal{N}_{b} \cup \mathcal{N}_{c}$, that is:

$$
\text { Minimize } Z(u)=\sum_{w \in W} g_{w} u_{w},
$$

which is the objective function of our model, where $u_{w}$ are the travel times at $\mathcal{N}_{a} \cup \mathcal{N}_{b} \cup$ $\mathcal{N}_{c}$, and $\left\{g_{w}\right\}_{\omega \in W}$ is the origin-destination demand matrix.

\subsection{Building the cost function}

The mathematical modelling of the set of highway corridors, which defines the new transport network $\mathcal{N}_{b}$ and existing highway network $\mathcal{N}_{a}$, is carried out through a set of $\widehat{n}=n+\tilde{n}$ 


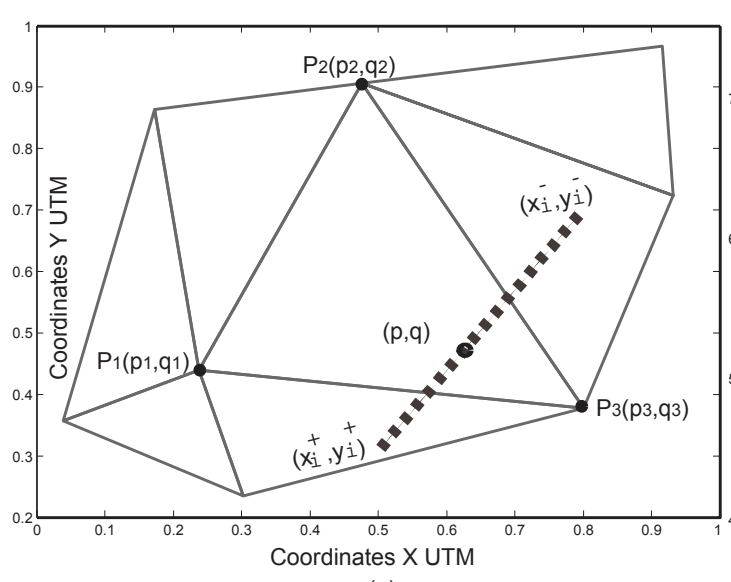

(a)

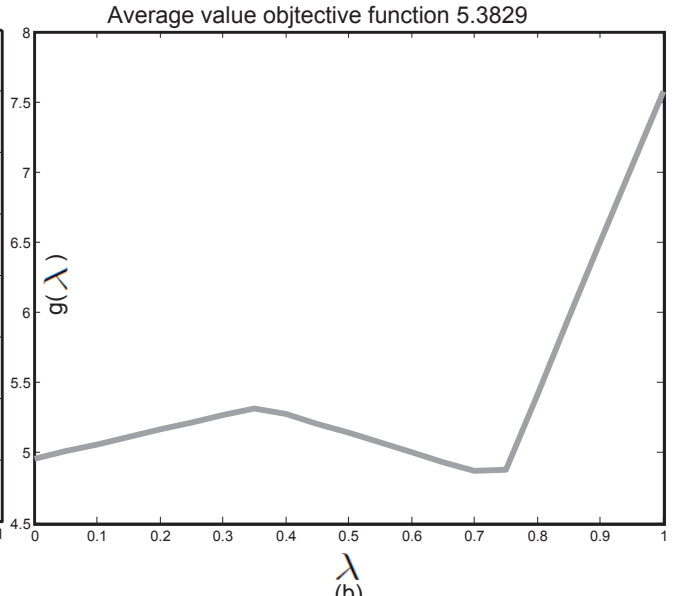

(b)

Figure 1: Cost function for a highway: (a) triangularization of study region; (b) construction cost for each linear unit

segments (where $n$ is the number of new highway corridors and $\tilde{n}$ is the number of existing highway network), defined by their endpoints. We consider that the segment linking endpoints is an approximation to the alignment it is intended to build. Thus, the model seeks to locate these new endpoints for the new highway corridors and the upper level variables are their coordinates $\left(x_{i}, y_{i}\right) ; i=1, \ldots n$. The model adds $n$ highway corridors.

In order to choose the best location for the new corridors, in addition to other factors, we need to consider the cost of land, the cost of bridges, earth movement, environmental cost and the type of soil in the different areas involved. In this section we explain how to incorporate all these factors to build the total cost function. The costs involved in the construction of a highway vary discontinuously with the terrain. Towns and cities, rivers, roads, and mountains appear and must be taken into account. Geographic Information Systems (GIS) provide the necessary information to estimate the construction costs of a linear unit of road at each point $(p, q)$ of the region of interest. In order to build the unit function $C(p, q)$, we consider a set of $m$ points $P_{j}=\left\{\left(p_{j}, q_{j}\right)\right\}_{j=1}^{m}$ covering regularly the area of interest and with the help of these GIS we estimate the construction costs per linear unit of highway at each point $C_{j}=C\left(p_{j}, q_{j}\right) j=1, \ldots, m$. These costs are the sum of the purchase of land, paving, earth movement, building junctions if there are intersecting roads, and building bridges if there are rivers, etc. The cost function $C\left(p_{j}, q_{j}\right)$, can incorporate information about especial or forbidden areas such as national parks, etc, by assigning higher cost values to the corresponding points.

Next, we proceed as follows. First, a triangularization of the study region based on the sample points $P_{j}$ is done [see Figure $1(\mathrm{a})$ ]. Next, to calculate the cost $C(p, q)$ at an arbitrary point $(p, q)$, we express the point $(p, q)$ as a linear convex combination of the vertices, i.e, $(p, q)=\sum_{k=1}^{3} \beta_{k}\left(p_{k}, q_{k}\right)$ with $\beta_{k} \geq 0$ with $k=1,2,3$ and $\beta_{1}+\beta_{2}+\beta_{3}=1$ 
and assign to this interior point the cost $C(p, q)=\sum_{i=1}^{3} \beta_{k} C_{k}$, where $C_{k} i=1,2,3$ are the costs associated with the vertices of the corresponding triangle where the point $(p, q)$ is located.

In this paper, each highway corridor $i$ is defined by a segment with endpoints $\left(x_{i}^{+}, y_{i}^{+}\right)$ and $\left(x_{i}^{-}, y_{i}^{-}\right)$[see Figure 1(a)]. To refer to an arbitrary point on the corridor a parameter $\lambda$ is used, such that its coordinates are $\left((1-\lambda) x_{i}^{+}+\lambda x_{i}^{-},(1-\lambda) y_{i}^{+}+\lambda y_{i}^{-}\right)$. In this way $\lambda=0$ represents the corridor starting point, and $\lambda=1$, the corresponding endpoint. Figure 1 (b) shows the function $g(\lambda)=C\left((1-\lambda) x_{i}^{+}+\lambda x_{i}^{-},(1-\lambda) y_{i}^{+}+\lambda y_{i}^{-}\right)$with $\lambda \in[0,1]$ which represents the construction cost for each linear unit at an arbitrary point of the highway.

The mean cost of the highway corridor is calculated by the integral $\int_{0}^{1} g(\lambda) \mathrm{d} \lambda$. Finally, the cost of a corridor can be evaluated by multiplying the total length by the mean construction cost of each linear unit, which gives the following expression:

$$
\begin{aligned}
K_{i}\left(x_{i}^{+}, x_{i}^{-}, y_{i}^{+}, y_{i}^{-}\right)= & \sqrt{\left(x_{i}^{-}-x_{i}^{+}\right)^{2}+\left(y_{i}^{-}-y_{i}^{+}\right)^{2}} \\
& \times \int_{0}^{1} C\left((1-\lambda) x_{i}^{+}+\lambda x_{i}^{-},(1-\lambda) y_{i}^{+}+\lambda y_{i}^{-}\right) d \lambda
\end{aligned}
$$

The total cost of the $n$ corridors then becomes $K(x, y)=\sum_{i=1}^{n} K_{i}\left(x_{i}^{+}, x_{i}^{-}, y_{i}^{+}, y_{i}^{-}\right)$ where $x=\left(x_{1}^{-}, x_{1}^{+}, x_{2}^{-}, x_{2}^{+}, \ldots, x_{n}^{-}, x_{n}^{+}\right)$and $y=\left(y_{1}^{-}, y_{1}^{+}, y_{2}^{-}, y_{2}^{+}, \ldots, y_{n}^{-}, y_{n}^{+}\right)$are the vectors of coordinates of the highways endpoints.

Once the cost function $K(x, y)$ has been built, we can write the budgetary constraint, which restricts the total cost of the located infrastructures, as:

$$
K(x, y) \leq B_{0}
$$

where $B_{0}$ is the available budget.

In conclusion, instead of using the GIS information directly in the model to evaluate the cost function, it has been used to construct a $2 \mathrm{D}$ piecewise linear function for interpolating the cost function. This has been done to reduce the computational burden.

\subsection{Travel time}

In this section the mechanisms for identifying the optimal route taken by a user and the total travel time in the transport facility system with new added highway corridors, $\mathcal{N}_{a}$ $\cup \mathcal{N}_{b} \cup \mathcal{N}_{c}$, are developed. The model considers that the users travel at a constant speed $v$ in $\mathcal{N}_{a} \cup \mathcal{N}_{b}$ and at a speed $w<v$ in the access network $\mathcal{N}_{c}$. Figure 2 illustrates different speeds considered in the proposed continuous model. In $\mathcal{N}_{a} \cup \mathcal{N}_{b}$ highways (black lines) the speed is $v$, and in the rest (grey shadowed zone) that corresponds to the access network 


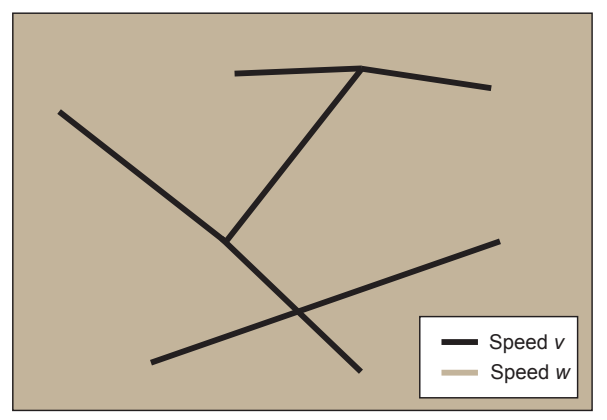

Figure 2: Illustration of the different speeds considered in the proposed model

zone, the speed is $w<v$. The expanded highway network $\mathcal{N}_{a} \cup \mathcal{N}_{b}$ is represented by the set of segments. The access network $\mathcal{N}_{c}$ (roads) allows direct travel between two points on the plane or input/exit at any point on the highways.

The travel time in network $\mathcal{N}_{a} \cup \mathcal{N}_{b} \cup \mathcal{N}_{c}$ is the sum of the access time and the travel time on the segments. The total travel time in the network is represented by $u=u_{a}+u_{b}+u_{c}$, where $u_{a}$ is the travel time in network $\mathcal{N}_{a}, u_{b}$ is the travel time in network $\mathcal{N}_{b}$ and $u_{c}$ is the travel time in network $\mathcal{N}_{c}$. To evaluate the objective function (5) travel times $u$ are calculated for each OD pair and all users are routed through the minimum travel time paths because we assume uncongested highways.

Figure 3 illustrates how the total travel time $u$ is calculated for a simple example, in which we have one origin city and three destination cities, three OD pairs $w=\{0 \rightarrow 1,0 \rightarrow$ $2,0 \rightarrow 3\}$, and $\mathcal{N}_{a} \cup \mathcal{N}_{b}$ consists of highways modelled by two segments (corridors 1 and 2 ). Figure 3 shows $\mathcal{N}_{a} \cup \mathcal{N}_{b} \cup \mathcal{N}_{c}$ in its upper left graph and three study cases corresponding to the minimum travel times $u$ calculated for each of the three OD pairs. In case 1 , the minimum travel time of pair $0 \rightarrow 1$ is $u_{1}=\frac{d_{1}}{w}$, where $d_{1}$ is the travelled distance and $w$ the speed; in case 2 , the minimum travel time of pair $0 \rightarrow 2$ is $u_{2}=\frac{d_{2}+d_{4}+d_{6}}{w}+\frac{d_{3}+d_{5}}{v}$, where $d_{1}$ to $d_{5}$ are the travelled distances in the different services and $w, v$ the associated speeds, and, in case 3 , the minimum travel time of pair $0 \rightarrow 3$ (end point of corridor 2 is above destination city 3 ) is $u_{3}=\frac{d_{2}}{w}+\frac{d_{7}}{v}$, where $d_{2}$ and $d_{7}$ are the travelled distances in the different services.

Therefore, a user to make a journey through $\mathcal{N}_{a} \cup \mathcal{N}_{b}$, must decide:

i) The entry/leaving points for each highway corridor.

ii) The set of highway corridors required to make the journey.

Decision i) consists of two subproblems, which are discussed in the following subsections. 


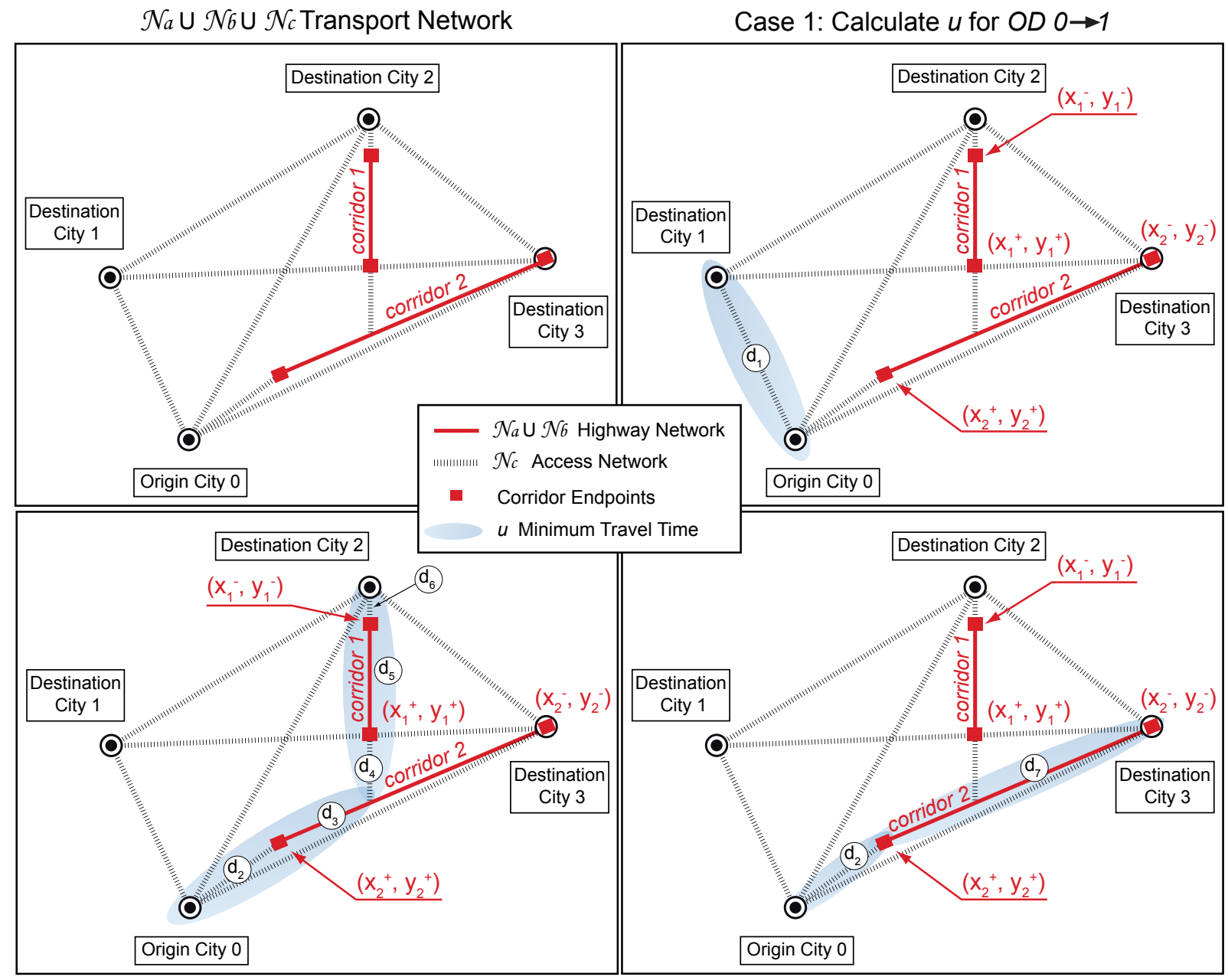

Case 2: Calculate $u$ for $O D O \rightarrow 2$

Case 3: Calculate $u$ for $O D O \rightarrow 3$

Figure 3: Ilustrative example

\subsubsection{Entry/leaving point from a centroid to a highway corridor.}

This problem is illustrated in Figure 4. It can be seen that users who decide to enter a transport facility have two possible optimal forms of entering the highway corridor depending on the travel direction. These directions are shown by superindices ${ }^{+}$and ${ }^{-}$.

To determine the entry point $\left(x^{+}, y^{+}\right)$to the highway corridor we must solve the following optimization problem:

$$
\begin{aligned}
& \text { Minimize } \\
& x^{+}, y^{+}, \lambda
\end{aligned}
$$




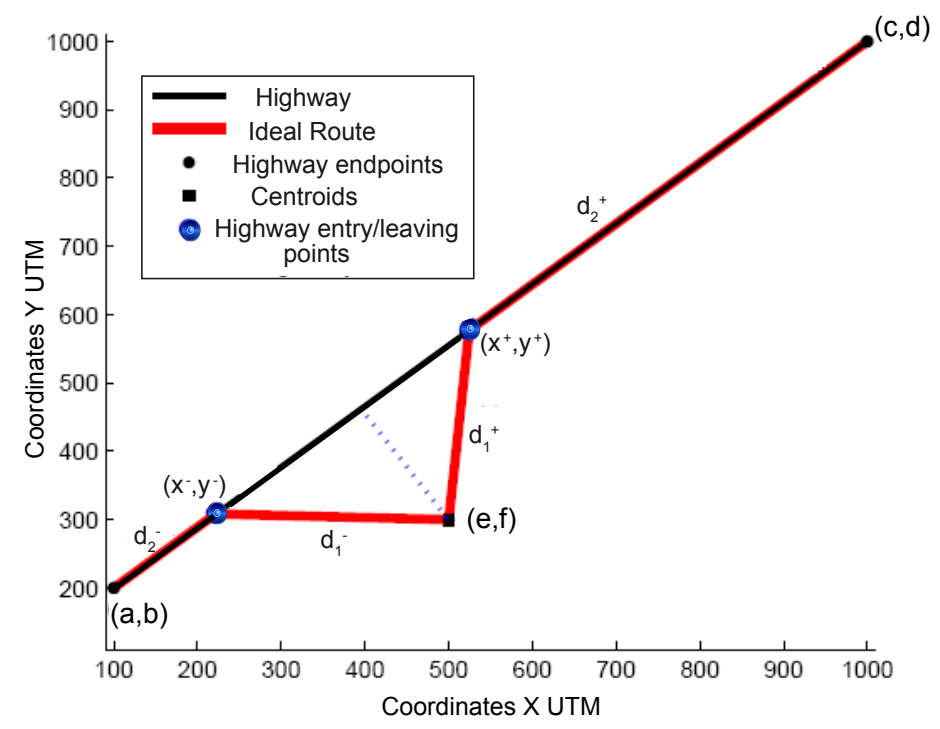

Figure 4: Entry/leaving points from a centroid $(e, f)$ to a highway corridor

$$
\begin{array}{ll}
\text { subject to }: & x^{+}=\lambda c+(1-\lambda) a \\
& y^{+}=\lambda d+(1-\lambda) b \\
& \lambda \in[0,1] .
\end{array}
$$

The objective function (8) represents the total travel time from $(e, f)$ up to $(c, d)$ passing troughout the entry point $\left(x^{+}, y^{+}\right)$. The first summand is the time taken from the origin $(e, f)$ to the point entering the transport system and the second summand is the time taken within the highway corridor to the endpoint $(c, d)$. Note that the Euclidean distance has been used. Replacing (9) and (10) into the objective function (8), this problem converts into a one-dimensional optimization problem in $\lambda$, whose solution is:

$$
\lambda_{*}^{+}=\max \left\{0, \min \left\{1, \lambda^{+}\right\}\right\},
$$

where

$$
\begin{aligned}
\lambda^{+} & =\frac{\sqrt{\left(v^{2}-w^{2}\right)}((c-a)(e-a)+(d-b)(f-b))}{\left((c-a)^{2}+(d-b)^{2}\right) \sqrt{\left(v^{2}-w^{2}\right)}}+ \\
& +\frac{w|(c-a)(f-b)-(d-b)(e-a)|}{\left((c-a)^{2}+(d-b)^{2}\right) \sqrt{\left(v^{2}-w^{2}\right)}}
\end{aligned}
$$

This provides a closed formula for $\lambda_{*}^{+}$, which when replaced into (9) and (10) provides closed expression for $x^{+}$and $y^{+}$.

Similarly, the optimum value for the entry point when travelling in the other direction is:

$$
\lambda_{*}^{-}=\min \left\{1, \max \left\{0, \lambda^{-}\right\}\right\},
$$


where

$$
\begin{aligned}
\lambda^{-} & =\frac{\sqrt{\left(v^{2}-w^{2}\right)}((a-c)(e-c)+(b-d)(f-d))}{\left((a-c)^{2}+(b-d)^{2}\right) \sqrt{\left(v^{2}-w^{2}\right)}}+ \\
& +\frac{w|(a-c)(f-d)-(b-d)(e-c)|}{\left((a-c)^{2}+(d-b)^{2}\right) \sqrt{\left(v^{2}-w^{2}\right)}}
\end{aligned}
$$

\subsubsection{Entry/leaving points between two highway corridors}

To illustrate this, consider the example with two highways shown in Figure 5 . The intended journey is from point $\left(a^{\prime}, b^{\prime}\right)$ of one highway to point $(c, d)$ of the other. Figure 5 illustrates the mathematical model, where $\mathcal{N}_{a} \cup \mathcal{N}_{b}$ is formed by highway corridors modelled in two segments. Users can enter or leave these infrastructures at any point. If a user wishes to go from point $\left(a^{\prime}, b^{\prime}\right)$ to $(c, d)$ using $\mathcal{N}_{a} \cup \mathcal{N}_{b}$ he will cover a distance $d_{1}$ on the first highway at a speed $v$, he will leave it at the node $\left(x^{\prime}, y^{\prime}\right)$ and will then travel at a speed $w$ for a distance $d_{2}$ to the access node $(x, y)$ of the second highway, completing the journey by travelling a distance $d_{3}$ on the second highway at a speed $v$. The total travel time in the network is calculated using the expression:

$$
u=\frac{d_{1}+d_{3}}{v}+\frac{d_{2}}{w} .
$$

Users will take the minimum travel time and so they will choose transfer points (exit and entry) $\left(x^{\prime}, y^{\prime}\right)$ and $(x, y)$ so that the total time $\frac{d_{1}+d_{3}}{v}+\frac{d_{2}}{w}$ taken on the journey is minimized; that is,

$$
\begin{array}{cc}
\text { Minimize } & \frac{\sqrt{\left(x^{\prime}-a^{\prime}\right)^{2}+\left(y^{\prime}-b^{\prime}\right)^{2}}}{v}+\frac{\sqrt{\left(x-x^{\prime}\right)^{2}+\left(y-y^{\prime}\right)^{2}}}{w}+\frac{\sqrt{(c-x)^{2}+(d-y)^{2}}}{v} \\
\text { subject to }: x^{\prime}, y^{\prime}, \lambda, \lambda^{\prime} & x=\lambda c+(1-\lambda) a \\
& y=\lambda d+(1-\lambda) b \\
& x^{\prime}=\lambda^{\prime} c^{\prime}+\left(1-\lambda^{\prime}\right) a^{\prime} \\
& y^{\prime}=\lambda^{\prime} d^{\prime}+\left(1-\lambda^{\prime}\right) b^{\prime} \\
& \lambda, \lambda^{\prime} \in[0,1] .
\end{array}
$$

Substituting the restrictions (18)-(21) associated with $x, x^{\prime}, y, y^{\prime}$ in the objective function, problem (17) becomes a generic expression of the type:

$$
\begin{gathered}
\underset{\lambda, \lambda^{\prime}}{\text { Minimize }} \quad f\left(\lambda, \lambda^{\prime}\right) \\
\text { subject to : } \quad \lambda, \lambda^{\prime} \in[0,1] .
\end{gathered}
$$




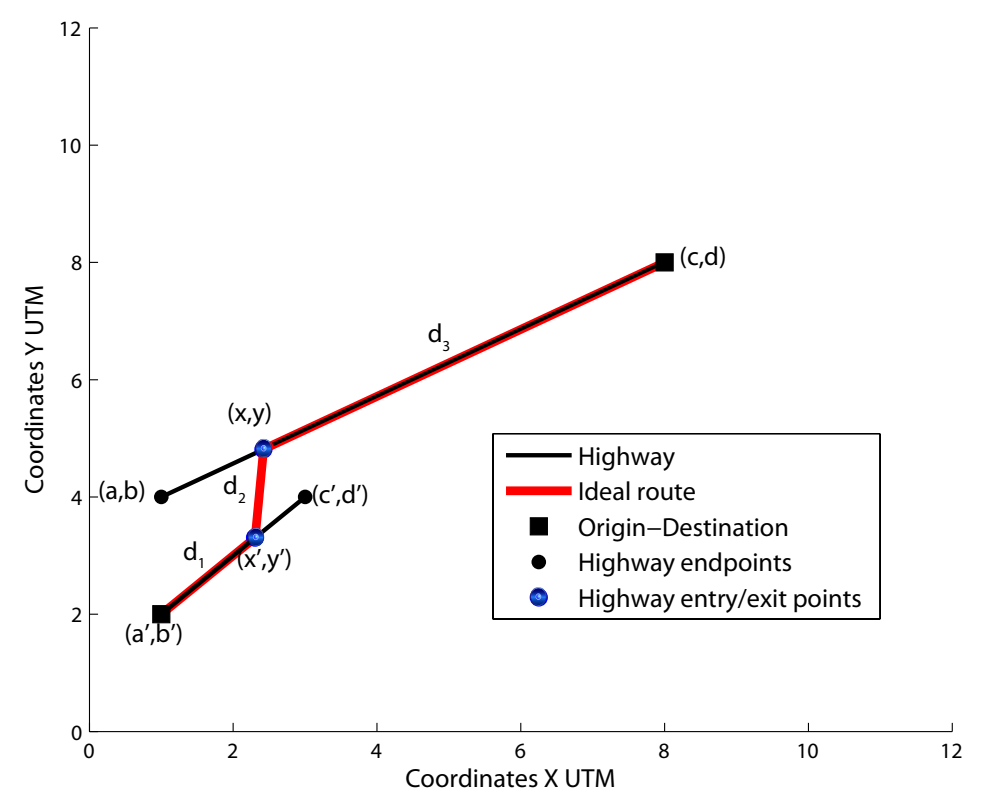

Figure 5: Entry/leaving points two highway corridors

The aim of the following remarks is to express problem (23)-(24) in two variables as an one dimensional optimization problem. Bellman's optimality principle applied to this problem states that the optimal path from $\left(a^{\prime}, b^{\prime}\right)$ to $(c, d)$ is made up of optimal subpaths, and so, if the exit point of the first highway $\left(x^{\prime}, y^{\prime}\right)$ were known, the entry point to the second highway $(x, y)$ must be optimal. Thus if $\lambda^{\prime}$ were known we could use the formula (13) and (15) to obtain the optimal value $\lambda$ as

$$
\begin{aligned}
\lambda\left(\lambda^{\prime}\right) & =\max \left\{0, \min \left\{1, \frac{\left((c-a)\left(x^{\prime}\left(\lambda^{\prime}\right)-a\right)+(d-b)\left(y^{\prime}\left(\lambda^{\prime}\right)-b\right)\right.}{\left((c-a)^{2}+(d-b)^{2}\right)}+\right.\right. \\
& \left.\left.+\frac{w\left|(c-a)\left(y^{\prime}\left(\lambda^{\prime}\right)-b\right)-(d-b)\left(x^{\prime}\left(\lambda^{\prime}\right)-a\right)\right|}{\left((c-a)^{2}+(d-b)^{2}\right) \sqrt{\left(v^{2}-w^{2}\right)}}\right\}\right\},
\end{aligned}
$$

where

$$
\begin{gathered}
x^{\prime}\left(\lambda^{\prime}\right)=\lambda^{\prime} c^{\prime}+\left(1-\lambda^{\prime}\right) a^{\prime} \\
y^{\prime}\left(\lambda^{\prime}\right)=\lambda^{\prime} d^{\prime}+\left(1-\lambda^{\prime}\right) b^{\prime} .
\end{gathered}
$$

Replacing (25) into the one dimensional problem (23)-(24) the problem to be solved becomes:

$$
\begin{gathered}
\underset{\lambda^{\prime}}{\operatorname{Minimize}} g\left(\lambda^{\prime}\right)=f\left(\lambda\left(\lambda^{\prime}\right), \lambda^{\prime}\right) \\
\text { subject to }: \lambda^{\prime} \in[0,1] .
\end{gathered}
$$




\subsubsection{Highway corridors required to make the journey}

To address problem ii) the so-called strategy graph has been developed, with the purpose of calculating the optimal route for a specified user of network $\mathcal{N}_{a} \cup \mathcal{N}_{b} \cup \mathcal{N}_{c}$. This graph is created for each origin of the OD matrix and contains, as nodes, one origin centroid $\mathrm{O}$, all the destination centroids for origin $\mathrm{O}$ and all the highway corridors by duplicated, one for each direction. The links model the different choices that a user can make. A link joining nodes $A_{1}^{p}$ and $A_{2}^{q}$ implies that a traveller would use highway corridor $A_{1}$ in the direction indicated by $p \in\{+,-\}$ and would leave it to continue the journey by highway corridor $A_{2}$ in the direction $q \in\{+,-\}$. The links joining the two directions $\left(A_{1}^{+} \rightarrow A_{1}^{-}\right)$of a single highway corridor have been omitted, since these links never form part of the optimal user strategy. The strategy graph is thus a complete graph in which links joining the same highway corridor in opposite directions have been removed. The direct links between centroids represent routing in roads that are not highways in the expanded network $\mathcal{N}_{a} \cup \mathcal{N}_{b}$. A path in the strategy graph defines the set and the order in which the different elements in the network must be used.

To illustrate the concept of strategy graph, consider the graph in Figure 6 for the two-highway case and a demand pair $w=(\mathrm{O}, \mathrm{D})$. The path $\mathrm{O} \rightarrow A_{1}^{+} \rightarrow A_{2}^{-} \rightarrow \mathrm{D}$ means that after entering highway corridor $A_{1}$ in the direction + , and having travelled along the section, the users choose to leave it and enter highway corridor $A_{2}$ in the specific direction - , going next to the destination centroid $\mathrm{D}$. This path defines which highway corridors are used, in which direction and in which order so as to complete the journey O-D.

The links of the strategy graph are divided into three types (see Figure 6), depending on how the costs are calculated:

1. Links between centroids. The travel time of this type of link is: $t_{O D}=\frac{d_{O D}}{w}$ were $d_{O D}$ is the Euclidean distance between centroids. Figure 6 shows one link $(\mathrm{O} \rightarrow \mathrm{D})$ of this type and $w$ is the link speed. It is denoted by $t_{O D}$ and is illustrated in Figure 7(a).

2. Links adjacent to centroids. The cost of this type of link (travel time) is the travel time from the centroid to the entry/exit point. The optimal point of this entry is calculated by one of the values $\lambda_{*}^{+}, \lambda_{*}^{-}$(see (12) and (14)). Note that we are calculating the optimal strategy from the fixed origin $\mathrm{O}$, and that entries to all highway corridors in both directions have been calculated in subsection 2.3.1.

The number of entries from centroid $\mathrm{O}$ is $2 \widehat{n}$ (the highway can be used in anyone of the two directions), where $\widehat{n}$ is the number of transport highway corridors in network $\mathcal{N}_{a} \cup \mathcal{N}_{b}$. By $\mathcal{N}_{I}=\{1, \ldots, 2 \widehat{n}\}$ the set of these entries are indicated and by $t_{(O, i)}$, $\forall i \in \mathcal{N}_{I}$ the travel time required to entering the highway corridor are indicated.

Likewise, the formula developed in subsection 2.3.1 allows us the calculation of the exit point and the exit travel time from a highway corridor to a destination $\mathrm{D}$. It is denoted by $t_{r D}$ and is illustrated in Figure $7(\mathrm{~b})$. The difference with respect to the previous case is that this cost involves the time used within the highway corridor 


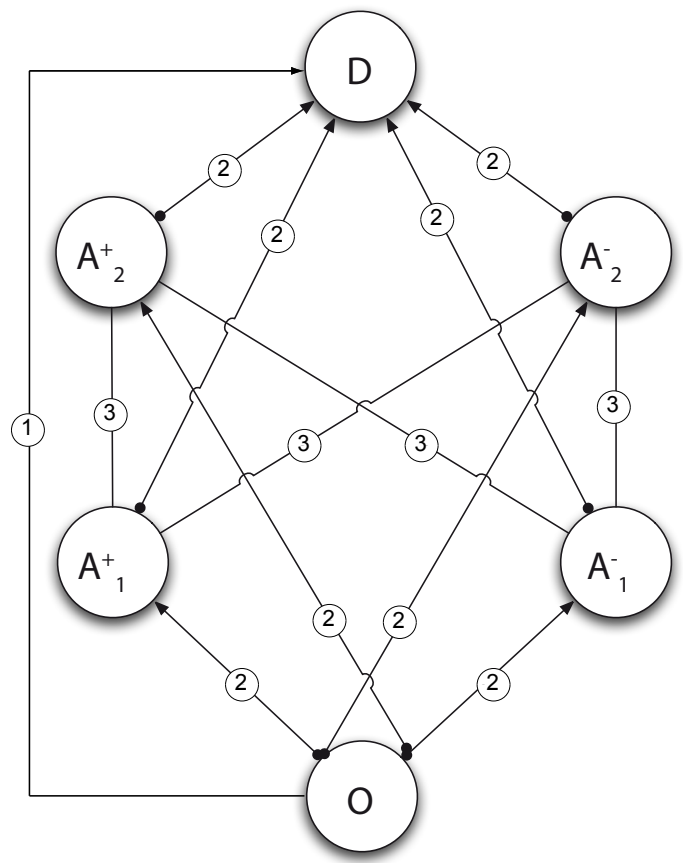

Figure 6: Strategy graph for two highway corridors showing its different link types

and also the time from there to destination D. Figure 6 shows eight links of this type $\left(\mathrm{O} \rightarrow A_{1}^{+}, \mathrm{O} \rightarrow A_{2}^{+}, \mathrm{O} \rightarrow A_{1}^{-}, \mathrm{O} \rightarrow A_{2}^{-}, A_{1}^{+} \rightarrow \mathrm{D}, A_{2}^{+} \rightarrow \mathrm{D}, A_{1}^{-} \rightarrow \mathrm{D}, A_{2}^{-} \rightarrow\right.$ D).

3. Links between highway corridors. In this case, the costs from the entry to the first highway corridor to the entry into the second are considered. It is denoted by $t_{r s}$ and is illustrated in Figure $7(\mathrm{c}) t_{r s}=\frac{d_{1}}{v}+\frac{d_{2}}{w}$. These costs are calculated approximately by solving the one dimensional optimization problem defined by (28)-(29). In this paper, the method based on a parabolic interpolation has been considered (see, for example, [3] and a detailed description of this method in [6]). Figure 6 shows four links of this type $\left(A_{1}^{+} \rightarrow A_{2}^{+}, A_{1}^{+} \rightarrow A_{2}^{-}, A_{1}^{-} \rightarrow A_{2}^{+}, A_{1}^{-} \rightarrow A_{2}^{-}\right)$.

Figure 8 shows Dijkstra's algorithm applied to the strategy graph.

This algorithm works with two node sets. The set of nodes $U$ contains in each iteration those nodes $r$ where the partial minimal path from the origin node $O$ has been determined. For node $r \in U, u(r)$ is the value of the travel time associated with the minimal path from $O$ to $r$. For the complementary node set $\mathcal{N}-U$, the minimal path through nodes in $U$ is known, and $h(s)$ with $s \in \mathcal{N}-U$ includes this value.

In Step 2 a new node $r \in \mathcal{N}-U$ is determined for which the minimal path has been found. In Step 3 the transit labels $h(s)$ adjacent to node $r$ are updated. 

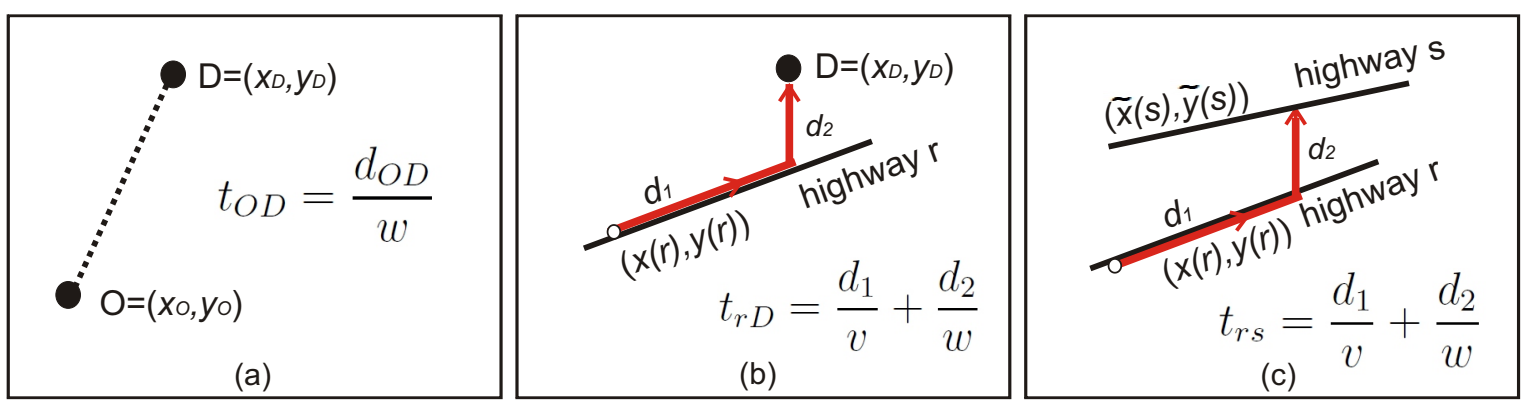

Figure 7: Cases of link cost in the strategy graph

The computational cost involved in applying Dijkstra's algorithm to the strategy graph is largely due to the computation of the link weightings, as in most of the links the problem of the optimization must be solved (28)-(29). This algorithm has the characteristic that the weight (travel time) is calculated when it is used. When the set of shortest paths to the destinations $\mathrm{W}$ is obtained (that is $W \subset U$ ) the algorithm stops. Possibly some of the links have not been considered and so the CPU time required to calculate these costs is saved.

In the case of congested networks, the algorithm described can be extended to flowdependent speeds $v$. The equilibrium traffic assignment models assume that travel times are independent of flow through the so-called link travel-time functions [19]. [5] describes a fixed point iteration process to solve this kind of model. In this approach, firstly a load for network demand is performed (network loading) from fixed link travel times and then new travel times are calculated for the new flows. This process is then iterated until a fixed point type solution is reached. This approach is applicable to the model described; firstly network loading would be performed as described for the uncongested case. In the second stage the average flows are calculated on this flow pattern and by a link travel time (for example from the Bureau of Public Roads (BPR) functions, [53] the new travel time in the highway section and thus the new average travel speeds $v$ in each highway with which to restart the procedure until a set convergence is reached. Note that in the case of congestion the speed on each highway is different.

\subsection{A multiobjective approach to handling uncertainty in de- mand}

The CNDP* problem is seen in the context of strategic planning. The estimated OD demand matrix may be inaccurate and affected by many factors not accounted for in the planning process. In the context of strategic planning it may be more suitable to consider a variety of scenarios and even a variety of different estimation techniques. This approach would lead to working with multiple OD demand matrices, where the goal is to 
Step 1. Initialization.

$U=\{O\}$.

Let $V=\{d \in \mathcal{O} \mid(O, D) \in W\} ; \mathcal{N}_{I}=\{1, \ldots, 2 \widehat{n}\}$ and $\mathcal{N}=\mathcal{N}_{I} \cup V$.

Let $u(O)=0, x(O)=x_{O}, y(O)=y_{O}$, where $\left(x_{O}, y_{O}\right)$ is the UTM coordinates of $O \in \mathcal{O}$.

Calculate $t_{O s}$ and let $(x(s), y(s))$ be the optimal entrance from origin $O$ to highway $s$ for all $s \in \mathcal{N}_{I}$; and for all $s \in \mathcal{N}-\{O\}$ compute.

$$
h(s)= \begin{cases}t_{O s} & s \in \mathcal{N}_{I} \\ \frac{d_{O s}}{w} & s \in V\end{cases}
$$

where $d_{O s}$ is the euclidean distance between origin $O$ and destination $s$.

Let $p(s)=0$ for all $s$.

Step 2. Expand $U$.

Let

$$
\begin{array}{r}
h(r)=\text { minimize }_{s \notin U} h(s) \\
r=\operatorname{Arg} \text { minimize }_{s \notin U} h(s)
\end{array}
$$

Take $U=U \cup\{r\}$ and $u(r)=h(r)$. If $V \subseteq U$, Stop.

Step 3. Update labels.

If $r \in V$ go to Step 2, otherwise $r \in \mathcal{N}_{I}$ and for all $s \notin U \cup\{\bar{r}\}$, where $\bar{r}$ is the same highway corridor $r$ but in the opposite direction to the original $r$. Calculate $t_{r s}$. Update $h(s) \leftarrow \min \left\{h(s), u(r)+t_{r s}\right\}$

If $h(s)=u(r)+t_{r s}$ take $p(s)=r$. Moreover if $s \in \mathcal{N}_{I}$ then update the entry to $s$ taking $x(s)=\tilde{x}$ and $y(s)=\tilde{y}$. Let $(\tilde{x}, \tilde{y})$ be its optimal entry. Go to Step 2.

Exit: the minimum path cost through the transport facility system from the origin $O$ to all the destination centroids, and this information is contained in the vector of variable $u$. The optimal strategy for the pair $(O, D) \in W$ is

$$
D \leftarrow p(D) \leftarrow p(p(D)) \leftarrow p(p(p(D))) \leftarrow \ldots \leftarrow O
$$

find a robust expansion of the highway network, i.e. one that is suited to the majority of scenarios defined by the set of OD matrices. Let $Z_{j}$ be the total travel time in the network for the OD matrix $j$, and $Z_{j}(u)$ means that this value depends on the travel time vector $u$ in the expanded network. The multiobjective extension of the CNDP* problem is formulated as follows:

$\left[\mathrm{MO}-\mathrm{CNDP}^{*}\right]$

$$
\underset{x, y}{\operatorname{Minimize}} \vec{Z}(u)=\left(Z_{1}(u), \cdots, Z_{k}(u)\right)
$$




$$
\begin{array}{cc}
\text { subject to: } \quad K(x, y) \leq B_{0} \\
u=H(x, y),
\end{array}
$$

The MO-CNDP* problem gives a set of nondominated solutions as the basis of a multicriteria decision analysis incorporating other factors not taken into account by the model. The result of the process would lead to a solution that could actually be put into effect.

\section{Application of Particle Swarm Optimization to bi- level continuous location model}

Particle Swarm Optimization (PSO, [39]) is a kind of random search algorithm that is based on the metaphors of social interaction and communications. PSO has been shown to be effective in solving difficult and complex optimization problems in a wide range of fields. In this paper, PSO is selected to generate an optimal network of highway corridors.

Firstly, the optimization problem (1)-(3) is reformulated as the unconstrained optimization problem, by the expression:

$$
\begin{aligned}
& \text { Minimize } \\
& \quad x, y
\end{aligned}
$$

where $\rho>0$ is a high weight value, $x$ and $y$ define the endpoint coordinates of the highway corridors and $Z(H(x, y))$ is described in subsections 2.1 and 2.3.

To simplify the notation, we denote the $4 \widehat{n}$ dimensional vector of decision variables by

$$
\mathcal{X}=\left(x_{1}^{+}, x_{1}^{-}, \cdots, x_{n}^{+}, x_{n}^{-}, y_{1}^{+}, y_{1}^{-}, \cdots, y_{n}^{+}, y_{n}^{-}\right)
$$

where $\widehat{n}=n+\tilde{n}$ ( $n$ is the number of new highway corridors and $\tilde{n}$ is the number of existing highway networks), and the penalty function by

$$
F(\mathcal{X})=Z(H(\mathcal{X}))+\rho \max \left\{0,\left(K(\mathcal{X})-B_{0}\right)\right\}^{2}
$$

where $K(\mathcal{X})$ takes into account only $n$ new highway corridors for construction cost.

The choice of the PSO method has been based on the following three key features of the model:

- Unconstrained nonlinear optimization and derivative-free methods. Problem (33) is an unconstrained nonlinear optimization model. Obtaining closed formulae for the derivatives of functions $Z(H(\mathcal{X}))$ is impossible because they are implicitly defined, or they do not exist and so any choice of a resolution method should be made from among the existing unconstrained nonlinear optimization derivative-free methods. 
- Computational cost. The computational cost of evaluating the budget constraint $K(\mathcal{X})$ (based on evaluating the two-variable interpolation function) and calculating total travel time for all OD pairs $u=H(\mathcal{X})$ is high, and this should be borne in mind and parallel computing strategies should be sought.

- The model presents multiple local optima.

PSO method uses collaboration among a population of simple search agents called particles (potential solutions) to find optima in some search space. In this paper, the Standard PSO $2011^{1}$ is applied, but includes parallel computing for the particle performance.

In every iteration, each particle is updated by following two "best" values. The first, the so-called previous best, is achieved by self experience (Step 3) and the second, the so-called best previous best, through the experience of a subset of particles (Step 4). The set of particles that informs a particle is called its neighbourhood. After finding these two values, the velocity and the position of particles will be updated in Step 5.

The convergence of the PSO algorithmn depends on its initialization. The initial corridors are generated randomly assuming an uniform distribution inside the search space.

The complete computational procedure of the Standard PSO algorithm for solving CNDP* model is summarized in Figure 9. The only modification is that this version includes parallel computing in Step 2 (Evaluation). In this step, the objective function is evaluated and it is the major computational cost. Therefore, the CPU time is improved meaningfully.

\subsection{Multiobjective Particle Swarm Optimization}

[11] present a proposal, called Multiobjective Particle Swarm Optimization (MOPSO), which allows the PSO algorithm to be able to deal with multiobjective optimization problems.

To describe briefly MOPSO, we will introduce the basic concepts for multiobjective optimization.

Let

$$
F_{j}(\mathcal{X})=Z_{j}(H(\mathcal{X}))+\rho \max \left\{0,\left(K(\mathcal{X})-B_{0}\right)\right\}^{2},
$$

be the penalty function for the OD matrix $j$, we define the multiobjective penalty function such as

$$
\vec{F}(\mathcal{X})=\left(F_{1}(\mathcal{X}), \cdots, F_{k}(\mathcal{X})\right)
$$

For two vectors $a, b \in \mathbb{R}^{k}$, we say that $a \leq b$ if $a_{j} \leq b_{j}$ for $j=1, \cdots, k$ and that $a$ dominates $b$ in $\mathbb{R}^{k}$ (denoted by $a \prec b$ ) if $a \leq b$ and $a \neq b$.

\footnotetext{
${ }^{1}$ SPSO 2011 has been put on line at the Particle Swarm Central [49].
} 
Figure 9: Standard PSO Algorithm for CNDP*

Step 1. Initialization. Initialize the parameters: number of iterations $(N)$, number of particles $(S)$, learning factor $(c)$, inertia weight $(w)$ and the average number of "informants" $(K)$. These parameters should be determined according to the variables of the problem. [10] suggests the values $S=40, \omega=\frac{1}{2 \log (2)} \simeq 0.721, c=1 / 2+\log (2) \simeq 1.193$ and $K=3$.

Initializate population with random positions and velocities.

$$
\begin{array}{r}
\mathcal{X}_{i j}^{0}=\operatorname{Rand}\left(\mathcal{X}_{j}^{m i n}, \mathcal{X}_{j}^{\max }\right), \\
v_{i j}^{0}=\operatorname{Rand}\left(\mathcal{X}_{j}^{\text {min }}-\mathcal{X}_{i j}^{0}, \mathcal{X}_{j}^{\text {max }}-\mathcal{X}_{i j}^{0}\right),
\end{array}
$$

where $\operatorname{Rand}\left(\mathcal{X}_{j}^{\min }, \mathcal{X}_{j}^{\max }\right)$ is a random number in $\left[\mathcal{X}_{j}^{\text {min }}, \mathcal{X}_{j}^{\text {max }}\right]$ drawn according to the uniform distribution. The parameters $\mathcal{X}_{j}^{\text {min }}$ and $\mathcal{X}_{j}^{\text {max }}$ are respectively lower and upper bounds of the decision variables. The subindex $i$ is associated with the ith particle and the subindex $j$ with the $\mathrm{j} t h$ dimension of the decision variable. Let $N_{i}^{0}$ be the set of neighbours of the particle $i$ at time $t=0$. Let $\widetilde{F}_{i}=+\infty$ and $t=1$.

Step 2. Evaluation (parallel computing). Evaluate the penalty function (35) for each particle $i$. That is,

$$
F_{i}^{t-1}=F\left(\mathcal{X}_{i}^{t-1}\right) \text { with } i=1, \cdots, S
$$

Step 3. Find the previous best. Find the previous best value for each particle $i$. If the current value $F_{i}^{t-1}$ of particle $i$ is better than its previous optimal value in history, then set the current previous optimum $p_{i}^{t-1}=\mathcal{X}_{i}^{t-1}$ as the new previous best value of particle $i$.

Step 4. Find the best previous best in the neighbourhood. Update the best of the best positions found up to now by informants as follows: Let $i^{\prime}=\underset{s \in N_{i}^{t-1}}{\operatorname{Arg} \operatorname{minimize}}\left\{F\left(p_{s}^{t-1}\right)\right\}$, if $F\left(p_{i^{\prime}}^{t-1}\right)$ is less than the current value $\widetilde{F}_{i}$, then $\widetilde{F}_{i}=F\left(p_{i^{\prime}}^{t-1}\right)$ and

$l_{i}^{t-1}=p_{i^{\prime}}^{t-1}$.

Step 5. Update the velocity and position. If $l_{i}^{t-1} \neq p_{i}^{t-1}$ then set $c_{i}^{t-1}=c$; otherwise $c_{i}^{t-1}=\frac{3 c}{4}$. Let $G_{i}^{t-1}=\mathcal{X}_{i}^{t-1}+$ $c_{i}^{t-1} \cdot\left(\frac{p_{i}^{t-1}+l_{i}^{t-1}-2 \mathcal{X}_{i}^{t-1}}{3}\right)$ and let $\tilde{\mathcal{X}}_{i}^{t-1}$ be a random point in the hyper-sphere with centre $G_{i}^{t-1}$ and radius $\left\|G_{i}^{t-1}-\mathcal{X}_{i}^{t-1}\right\|$.

Step 5.1. Update the particle velocity for each particle $i$ according to the following equation

$$
v_{i}^{t}=w \cdot v_{i}^{t-1}+\widetilde{\mathcal{X}}_{i}^{t-1}-\mathcal{X}_{i}^{t-1}
$$

Step 5.2. Update the particle's position

$$
\mathcal{X}_{i}^{t}=\mathcal{X}_{i}^{t-1}+v_{i}^{t}
$$

Step 6 Stopping criterion. If the number of iterations hold $t=N$, Stop; otherwise continue. If the current iteration is unsuccessful (no improvement of the best known fitness value), define new neighbourhoods $N_{i}^{t}$ (refer the reader to [9] for a description of the adaptive random topology); otherwise $N_{i}^{t}=N_{i}^{t-1}$. Let $t=t+1$ and go back to Step 2 .

Exit: the vector $l_{i^{\prime}}^{N-1}$ of the coordinates of the optimal highway corridor and its optimal value $Z^{*}=Z\left(l_{i^{\prime}}^{N-1}\right)$.

We say that a vector $\mathcal{X} \in \mathbb{R}^{4 n}$ is a nondominated solution with respect to the search 
space $\mathbb{R}^{4 n}$, if there does not exist another $\mathcal{X}^{\prime} \in \mathbb{R}^{4 n}$ such that $\vec{F}\left(\mathcal{X}^{\prime}\right) \prec \vec{F}(\mathcal{X})$. At the opposite, $\mathcal{X}$ is said to be dominated, if there exists $\mathcal{X}^{\prime} \in \mathbb{R}^{4 n}$ such that $\vec{F}\left(\mathcal{X}^{\prime}\right) \prec \vec{F}(\mathcal{X})$.

We say that a vector of decision variables $\mathcal{X}^{*} \in \mathbb{R}^{4 n}$ is Pareto optimal if it is nondominated with respect to $\mathbb{R}^{4 n}$. The Pareto optimal set $P^{*}$ is defined by:

$$
P^{*}=\left\{\mathcal{X} \in \mathbb{R}^{4 n} \mid \mathcal{X} \text { is Pareto optimal }\right\}
$$

and the Pareto front $\mathcal{F}_{P^{*}}$ is defined by:

$$
\mathcal{F}_{P^{*}}=\left\{\vec{F}(\mathcal{X}) \in \mathbb{R}^{k} \mid \mathcal{X} \in P^{*}\right\}
$$

The goal is to discover solutions that are not dominated by any other in the search space.

MOPSO explores Pareto dominance concepts in order to update the velocity. Based on this concept each particle could have different leaders. This set of leaders is stored in a repository, which contains the best nondominated solutions found. The velocity is updated as follows:

$$
v_{i}^{t}=w \cdot v_{i}^{t-1}+c_{1} \cdot \operatorname{Rand}(0,1)\left(p_{i}^{t-1}-\mathcal{X}_{i}^{t-1}\right)+c_{2} \cdot \operatorname{Rand}(0,1)\left(r_{i}^{t-1}-\mathcal{X}_{i}^{t-1}\right) .
$$

where $r_{i}^{t-1}$ is a particle from the repository, chosen as a guide to particle $i, \operatorname{Rand}(0,1)$ is a random number and $c_{1}$ and $c_{2}$ are parameters which indicate how much each component influences velocity. To udpate the previous best, Pareto dominance is applied to the current position $\mathcal{X}_{i}^{t-1}$ and $p_{i}^{t-1}$. If neither of them is dominated by the other, then it is randomly selected. The external repository consists of an archive controller and a grid. The first element decides whether a certain solution is added or not based on Pareto dominance, and the grid produces well-distributed Pareto fronts. At the end of the algorithm, the solutions in the repository are the final output.

\section{Numerical results}

In this section a set of numerical experiments are carried out with the aim of determining the suitability of the proposed models and analyzing the obtained solutions. The numerical experiments aim at the following goals:

i) Comparison of the CNDP* and DNDP methods. A case study has been performed in order to test the feasibility of the proposed methodology in real problems. In subsection 4.3, a numerical experiment illustrates the advantages of the proposed CNDP* versus the DNDP model by comparing both solutions obtained in the case study. This section also assesses the applicability of the multiobjective extension MO-CNDP* to the case study.

ii) Analysis of the computational cost. In subsection 4.2 the performance of the parallel computing of Standard PSO and MOPSO are analyzed. 


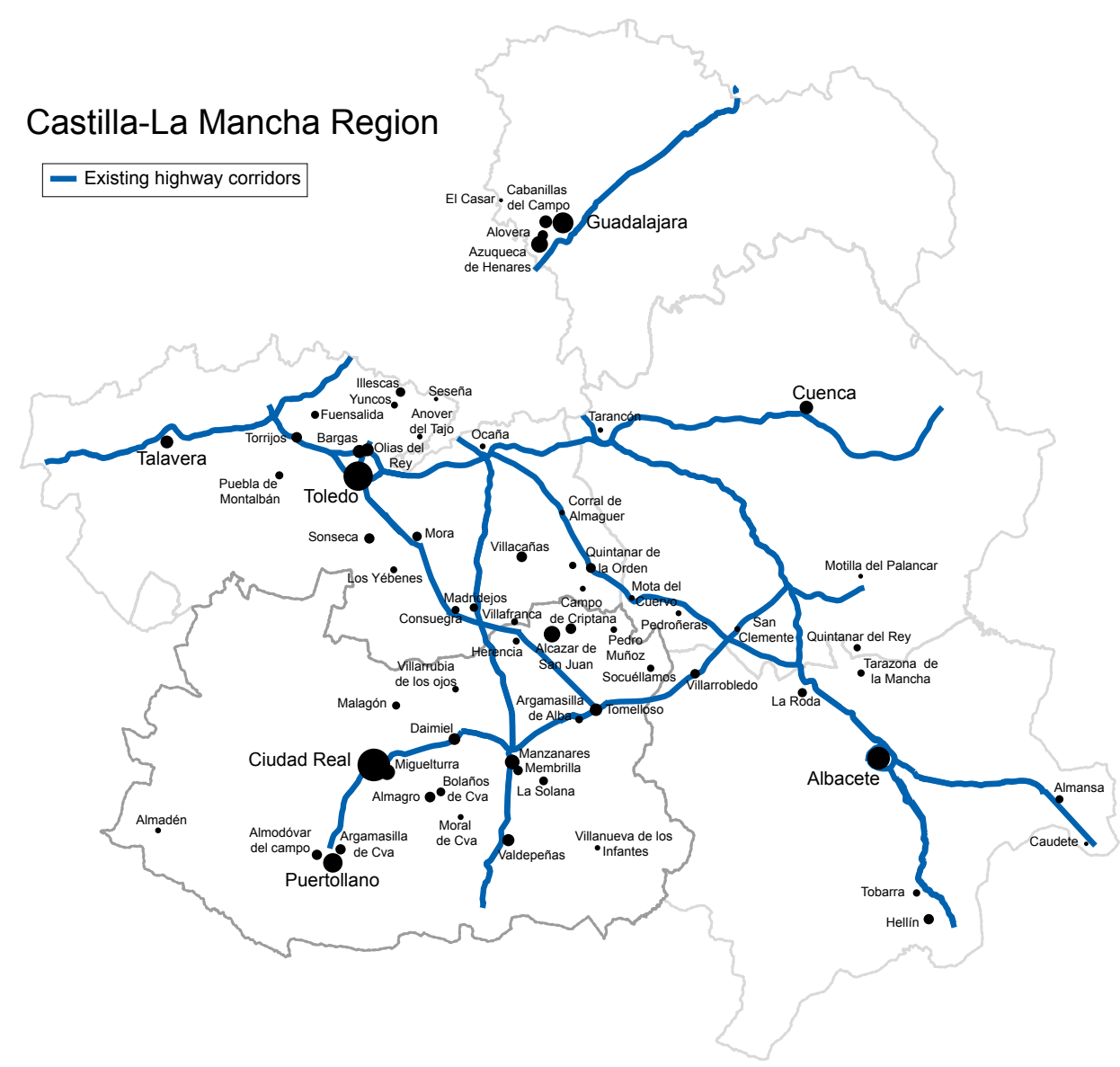

Figure 10: Castilla-La Mancha test problem

\subsection{Description of the numerical test}

The 67 towns with the highest populations in the region of Castilla-La Mancha (Spain) are considered. Figure 10 shows the 67 towns and the existing highway corridors in the region of Castilla-La Mancha. In the numerical tests the set of existing highways have been modelled by 8 segments.

The input of the problem ${ }^{2}$ consists of: (a) the coordinates UTM of the 67 towns considered, (b) two origin-destination matrices (c) existing highway endpoints and (d) data construction costs to be used in the cost function (6). In the study case a realistic analysis of the incurred costs is developed.

The first OD matrix consists of 1,626 OD pairs and has been estimated by the INE (National Institute of Statistics) based on surveys. The second OD matrix consists of

\footnotetext{
${ }^{2}$ The test problem is put on line in http://bit.ly/Xaq61f
} 
2,211 OD pairs and it has been adjusted using the following gravity model (see [48])

$$
g_{\omega} \propto P_{O} \cdot P_{D} \cdot \exp \left(-\beta d_{O D}\right)
$$

where the demand $g_{\omega}$ in the pair $\omega=(O, D)$ is proportional to population $P_{O}$ at the origin $O$ and to the population $P_{D}$ at the destination $D$ and $d_{O D}$ is the distance from $O$ to $D$. The parameter $\beta$ ensures a certain level of demand is met.

Table 2 shows the parameters of Standard PSO and MOPSO algorithms used in the numerical experiments.

Table 2: Parameters used by Standard PSO and MOPSO algorithms

\begin{tabular}{lcccccc}
\hline Algorithm & $\omega$ & $S$ & $c$ & $c_{1}$ & $c_{2}$ & $K$ \\
\hline Standard PSO & 0.721 & 40 & 1.193 & - & - & 3 \\
MOPSO & 0.730 & 40 & - & 1.496 & 1.496 & - \\
\hline
\end{tabular}

The MOPSO algorithm uses other parameters such as the repository size, set at 40, the number of grids for each dimension, set at 10, the leader section pressure parameter, set at 4 , and the extra (to be deleted) repository member selection pressure, set at 2 .

The penalty parameter $\rho$ should be chosen to be sufficiently large that the solution obtained satisfies the constraint $K(\mathcal{X}) \leq B_{0}$. Theorem 8.2 in [12], allows an analysis to be performed of the local sensitivity of the objective function to $\rho$. A value that is too low would lead to solutions whose construction costs would significantly exceed the available budget $B_{0}$. A priori it is not possible to choose a value of $\rho$ that is too high as this would cause problems of convergence in the algorithms. A heuristic rule to determine $\rho$ is based on homogenising the magnitudes used in the measuring of the transport and highway building costs. In this application we have measured the budget $K(\mathcal{X})$ in equivalent kilometres which can be built to an average construction cost.

The cost of an hour of transport is of the order of magnitude of $10^{1}$ Euros while for the construction of a kilometre of highway it is $10^{6}$ Euros. The total cost is of the order of $10 Z+10^{6} K(\mathcal{X})$ Euros. If we insist, for example, that building an extra 1 kilometres has a penalty of 100 times the equivalent cost in hours of travel, this would lead to an objective function $F=10 Z+10^{2} 10^{6} \max \left\{0, K(\mathcal{X})-B_{0}\right\}^{2}$. Finally, dividing the previous expression by 10 , we obtain the value for $\rho=10^{7}$ which was taken in all the trials for problems (33) and (39).

The Standard PSO, MOPSO and objective function (33) have been codified in MATLAB ([46], [49]) and all trials have been carried out with a Pentium computer with Intel(R) Core(TM) Quad CPU Q9550 $2.83 \mathrm{GHz}$ and 4 GB of RAM. 


\subsection{Analysis of the computational cost}

In this subsection the computational cost of Standard PSO and MOPSO algorithms versus strategies of parallel computing is analysed. This is a key issue in the applicability of the proposed methodology. Note that for each evaluation of the objective function and for each OD demand pair dozens of one-dimensional problems are solved in order to find the optimal strategy travel and associated travel time $u$. Moreover, the number of evaluations of the objective function is $N \cdot S$, where $S$ is the number of particles and $N$ the number of iterations. The result is a intensive computational task.

In this numerical experiment the Standard PSO and MOPSO algorithms uses the parameter $S=40$ and $n=2$ new highway corridors are added to the $\tilde{n}=8$ existing highway corridors.

The experiment consists of carrying out one main iteration of Standard PSO and MOPSO using 1,2,3 and 4 microprocessors. Table 3 shows the obtained CPU time.

Table 3: CPU time (in seconds) to carry out one main iteration

\begin{tabular}{crr}
\hline Microprocessors & Standard PSO & MOPSO \\
\hline 1 & 5,890 & 8,988 \\
2 & 4,050 & 4,614 \\
3 & 3,770 & 3,511 \\
4 & 4,017 & 3,757 \\
\hline
\end{tabular}

The numerical results shows that the parallelization strategy saves around $30 \% \mathrm{CPU}$ time for SPSO and around $60 \%$ CPU time for MOPSO.

The computational cost to evaluate $K(\mathcal{X})$ is around $13 \%$ of the total CPU to evaluate the objective function. The main cost is the computation of $H(\mathcal{X})$.

\subsection{A case study}

In this section the results with $\mathrm{CNDP}^{*}$ and DNDP test problems employing a new realistic cost function with CNDP* are compared. The two approaches have been used to minimize the total travel time by adding new highway corridors to the existing road network in Castilla-La Mancha. The budget $B_{0}$ allows us to expand the existing network around with $200 \mathrm{~km}$ of new highways.

For the DNDP we have considered that highways between populations centres with no more about $30 \mathrm{~km}$ apart can be built. This network contains 2,154 possible arcs and the result is a large scale mixed linear programming model with 111, 098 equations and 8,645,971 variables. The DNDP has been solved using branch-and-cut algorithms (performed in CPLEX 11 and integrated in GAMS 22.8). 


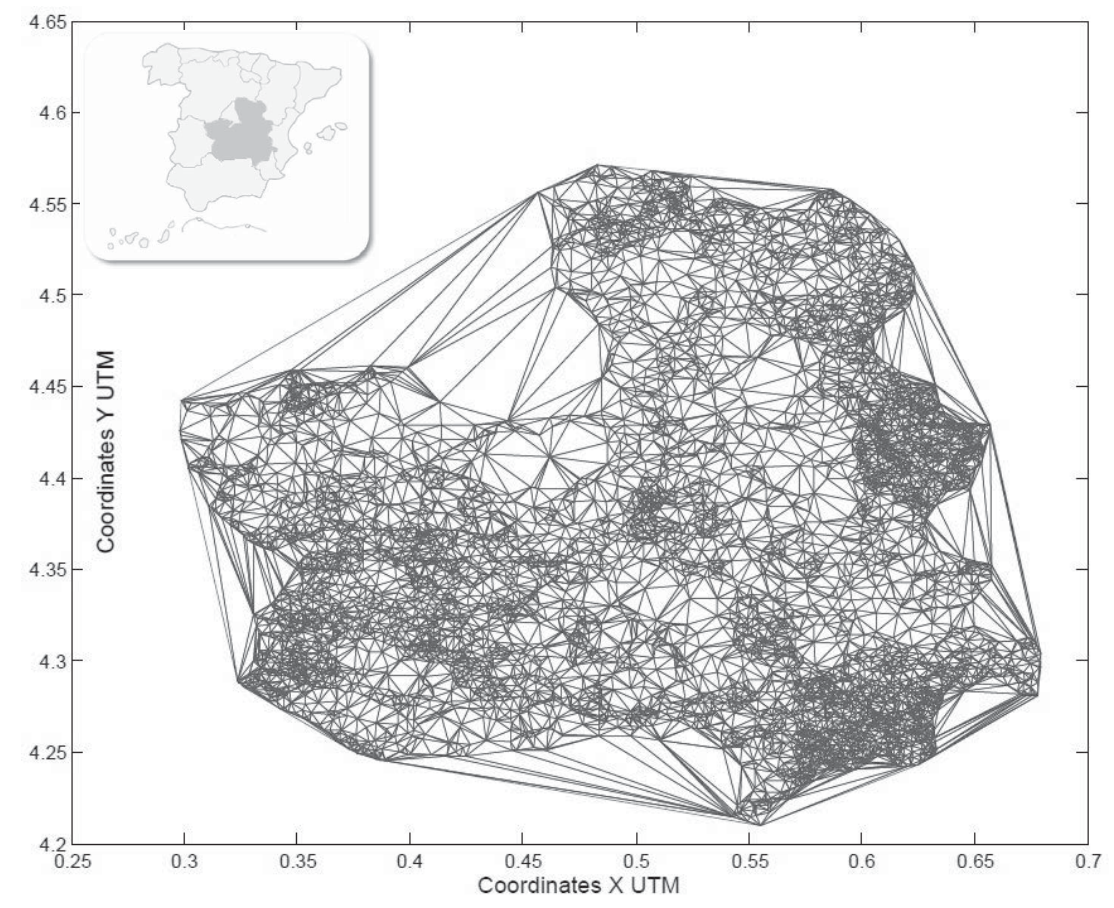

Figure 11: Triangularization of the study region

For the CNDP* we have assessed the construction costs on set of $m=9,728$ points in the region of Castilla-La Mancha, which represents a hundred million euros (due to rivers, mountains, soil types, etc.) They have been obtained after a costly evaluation process due to the difficulties in getting such a huge amount of information. Based on them, a linear interpolation function is used to derive the cost at any point. First a triangularization of the study region is carried out, generating 19,325 triangles as shown in Figure 11. From these data the piecewise linear interpolation function is obtained via the expression (6) of subsection 2.2 .

The computational cost is about 8 hours in the DNDP with a relative gap less than $3.5 \%$ and about 93 hours for the $\mathrm{CNDP}^{*}$ with parallel computing and using 4 cores (microprocessors).

Figure 12 compares the solution obtained with both approaches. CNDP* solution Figure 12(a) has thicknesses of the highway corridors proportional to flow.

The DNDP model locates 14 links small corridors for the given budget. The disadvantage of this solution is that it has a lot of disconnected components. The building of new highways is a very costly investment which must, in general, be done corridor by corridor. In highway network planning a very small number of corridors are analysed simultaneously. Solutions such as that found by the DNDP in the example suggest action 


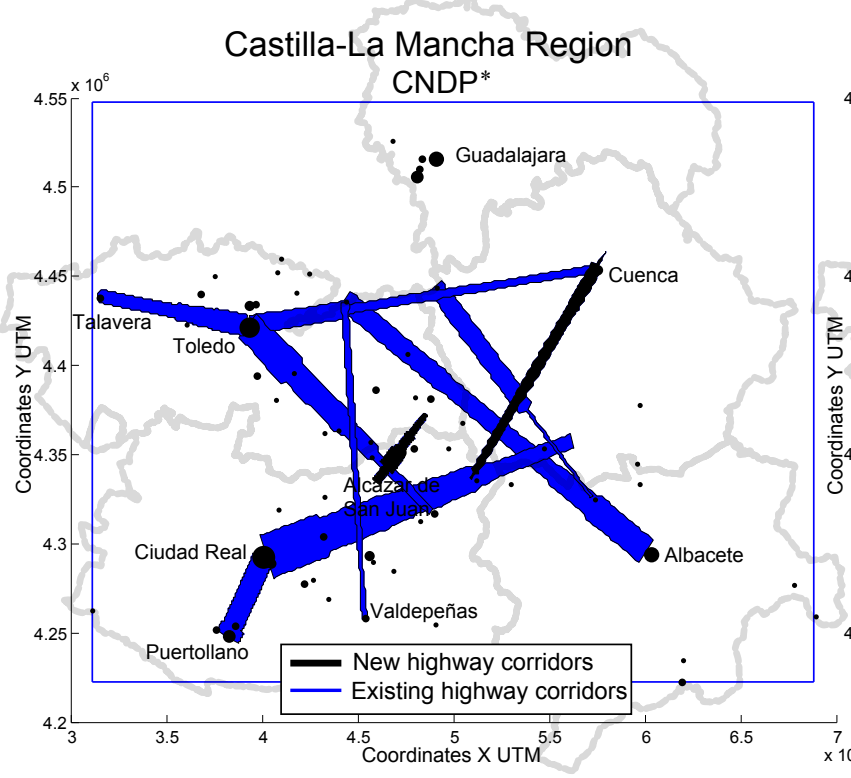

(a)

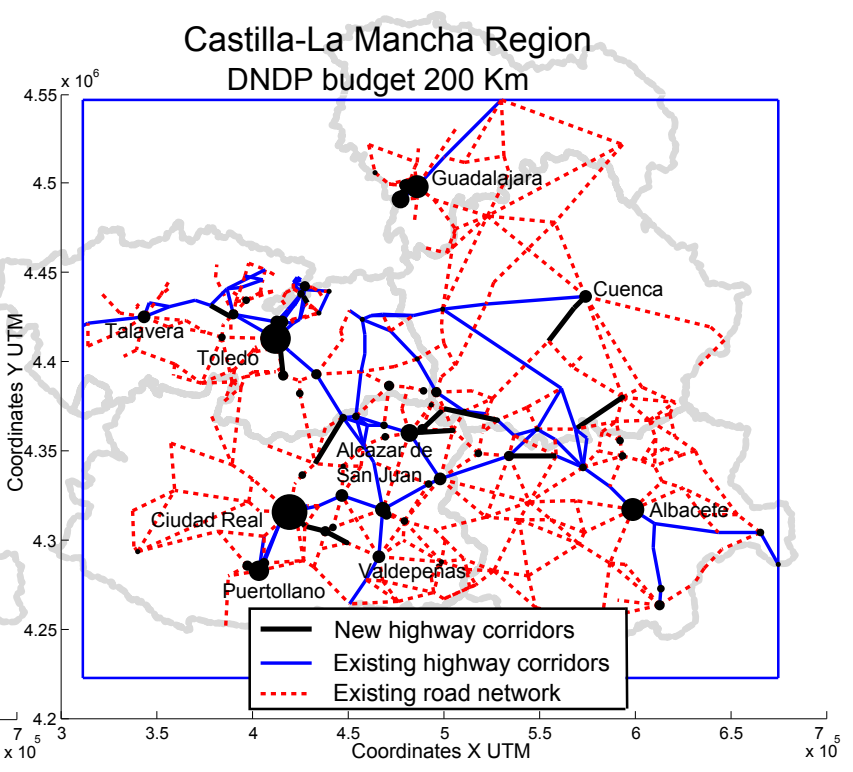

(b)

Figure 12: Illustrative comparing between DNDP and CNDP*

in 14 highway sections. This solution can provide information to the planner about flows in the study region but it does not completely define the corridor/s to be covered by the new highways in the same way as $\mathrm{CNDP}^{*}$ (see Figure 12). The solution obtained with

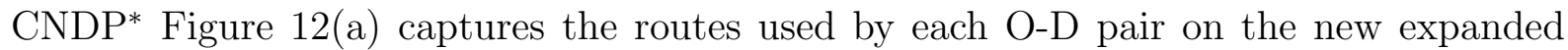
road network. The CNDP* model Figure 12(a) obtains 2 large corridors.

In conclusion, although DNDP uses a realistic routing mechanism and has the possibility of making fine adjustments to the speeds (link by link), the solution given by this method is unable to identify the corridors which would enhance the current network. The CNDP*, on the other hand, is based on many simplifications but it properly identifies the newly added corridors and estimates the redistribution of traffic flows.

The MO-CNDP* model allows uncertainty in demand to be addressed, working simultaneously with several OD demand matrices. The MO-CNDP* model is illustrated in the case study using two OD matrices. Figure 13 shows the Pareto front obtained from the MOPSO with $N=220$ iterations. The algorithm shows a behaviour which appears to converge to the optimal Pareto front but the results obtained with the CNDP* suggest the MOPSO algorithm should perform even more iterations.

Figure 14 shows two nondominant solutions (solution 1 and solution 2 shown in Figure 13) obtained with the MOPSO. The best solution with respect to the OD matrix estimated by the INE $\left(Z_{1}\right)$ agrees with the solution from the CNDP*.

In conclusion, the MO-CNDP* enriches the solution of the CNDP* problem, finding 


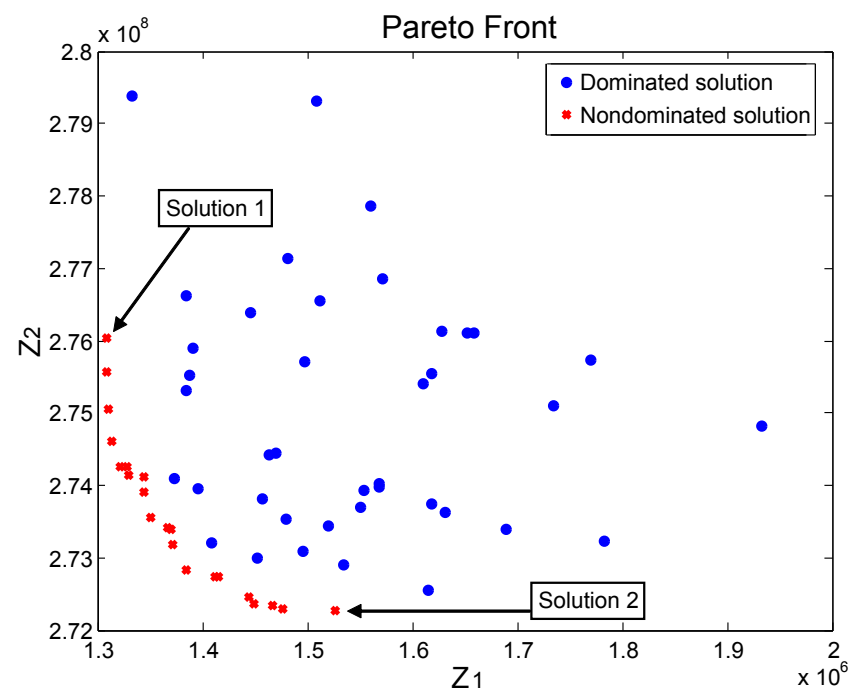

Figure 13: Pareto front obtained using MOPSO

a set of nondominated solutions. The price to be paid is a higher computational cost which, in the case of using a largest number of OD matrices, would make it unviable. In these cases the real problem could be addressed using the CNDP* for the expected OD matrix (interpreted as the synthesis of the set of OD matrices). This scheme, due to the fact that in the CNDP* model the flows are linear with respect to demand, is a stochastic programming approach which seeks to minimize total expected travel time.

\section{Conclusions and future research}

In this paper a bi-level continuous location model $\left(\mathrm{CNDP}^{*}\right)$ is proposed to expand an existing highway network. The upper level problem locates the highway alignments on a continuos space and the lower level computes the user's reaction by means of an implicit function which combines a unidimensional optimization method and a specialized form of shortest path algorithms.

The model locates a set of new highways with the aim of minimizing the total traveltime cost to satisfy all the origin-destination demand pairs in the study region while a given budget is satisfied. This goal may be of great concern from the user's viewpoint.

The cost function based on 2D-interpolation makes the problem highly flexible and allows a very accurate consideration of many factors such as land boundaries, environmentally sensitive regions, and topographic data. A second advantage with respect to DNDP is that the obtained solution is a complete set of alignments rather than a disjointed set of sections of several alignments. This has been illustrated in the numerical tests in section 

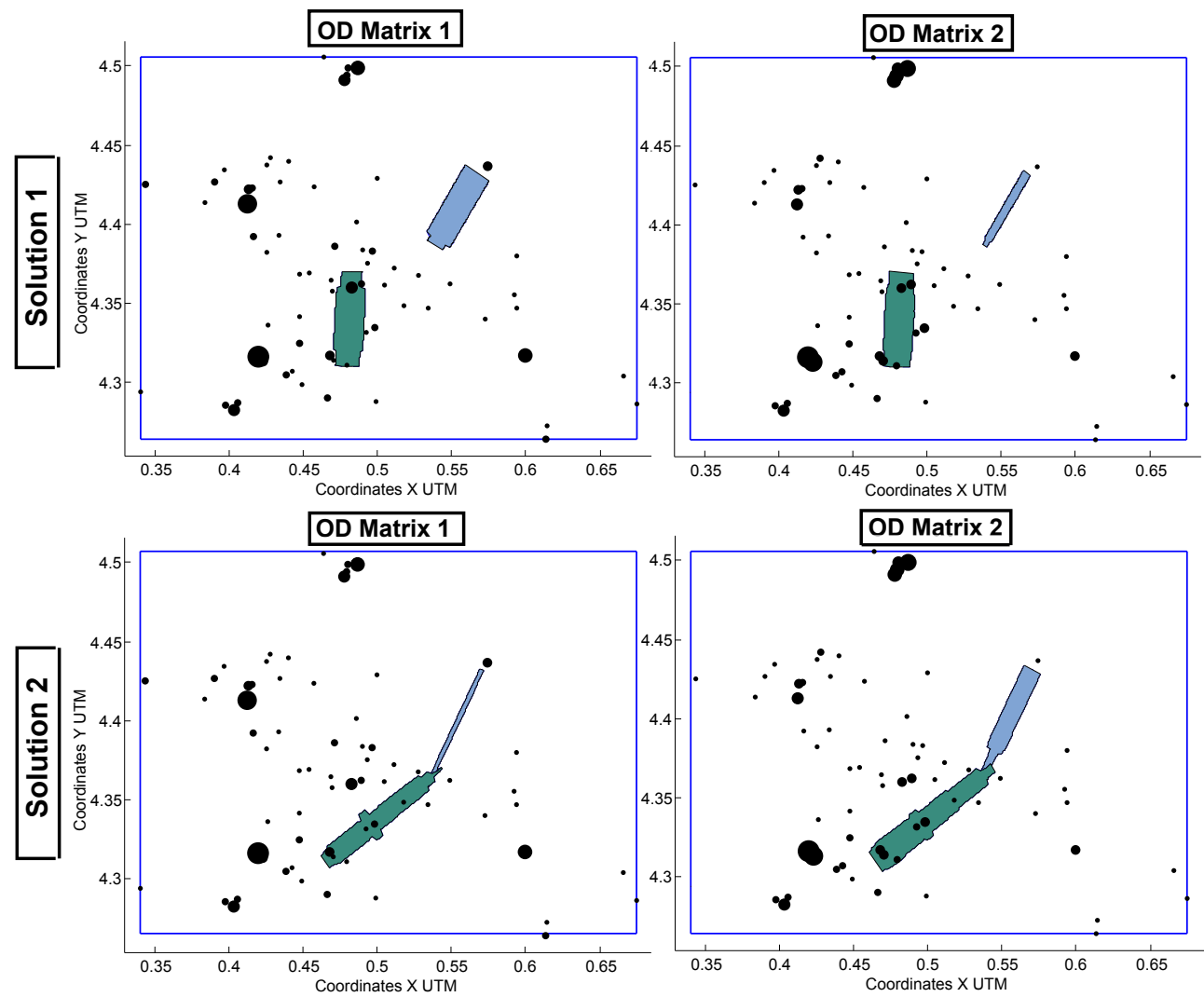

Figure 14: Nondominated solutions obtained by MOPSO

4.

The method presented here is particularly suitable for initial screening of the expansion of a highway network. The output from the model is aimed at producing the inputs necessary for the Highway Alignment Optimization stage such as the endpoints of a set of optimal alignments and the distibution of traffic flows in the expanded highway network.

The resulting bi-level model is complex and has required the development of a heuristic methodology for its solution. The complexity of the model severely limits the search algorithms that can be employed. The proposed PSO algorithm includes three main features: i) to take into account discountinous cost functions, ii) to search global solutions and iii) to allow for parallel computing.

The model is also tested in the region of Castilla-La Mancha (Spain) with heterogeneous land-use and mountainous terrain. The results demonstrate the convergence to a good solution. Moreover, the parallelization of Standard PSO allows the application of the proposed methods to real world problems.

The proposed methodology has been extended to a multiobjective scheme in order to 
address uncertainty in the OD matrix. A MOPSO was used to solve the problem and it was found that it is a procedure with a high computational cost. Future research will address the speeding up of the convergence of the MOPSO through the incorporation of new hybridization strategies such as that given by the Nelder-Mead method [16].

\section{Acknowledgements}

This research was supported by grant TRA2011-27791-C03-03 from Ministerio de Economía y Competitividad, Spain.

\section{References}

[1] E. Angulo, E. Castillo, R. García-Ródenas, and J. Sánchez-Vizcaíno. Determining Highway Corridors. Journal of Transportation Engineering, 137(5):557-570, 2011.

[2] A. Balakrishnan, T. L. Magnanti, and P. Mirchandani. Annotated Bibliographies in Combinatorial Optimization. M. DellÁmico, F. Maffioli, \& S. Martello. New York: Wiley, 1997.

[3] M. S. Bazaraa, H. D. Sherali, and C. M. Shetty. Nonlinear Programming: Theory and Algorithms. John Wiley \& Sons, New York, NY, second edition, 1993.

[4] D. E. Boyce and B.N. Janson. A Discrete Transportation Network Design Problem with Combined Trip Distribution and Assignment. Transportation Research, 14B:147-154, 1980.

[5] G. E. Cantarella. A General Fixed-Point Approach to Multimode Multi-User Equilibrium Assignment with Elastic Demand. Transportation Science, 31(2):107-128, 1997.

[6] E. Castillo, A. Conejo, P. Pedregal, R. García, and N. Alguacil. Building and Solving Mathematical Programming Models in Engineering and Science. Pure and Applied Series (PAMS), John Wiley \& Sons, Inc., New York, 2002.

[7] J. F. Cheng and Y. Lee. Model for Three-Dimensional Highway Alignment. ASCE Journal of Transportation Engineering, 132(12):913-920, 2006.

[8] E. P. Chew, C. J. Goh, and T. F. Fwa. Simultaneous Optimization of Horizontal and Vertical Alignments for Highways. Transportation Research Part B, 23(5):315-329, 1989.

[9] M. Clerc, Particle Swarm Optimisation. ISTE (International Scientific and Technical Ecyclopedia), 2006. 
[10] M. Clerc, Standard Particle Swarm Optimisation. From 2006 to 2011 http://clerc.maurice.free.fr/pso/SPSO_descriptions.pdf, 2012-09-13 version.

[11] C. Coello, G. Toscano, and M. Salazar. Handling multiple objectives with Particle Swarm Optimization. IEEE Transactions on Evolutionary Computation, 8(3):256279, 2004.

[12] A. Conejo, E. Castillo, R. Mínguez, and R. García-Bertrand. Decomposition Techniques in Mathematical Programming: Engineering and Science Applications. New York: Springer, Berlin, 2006.

[13] T.G. Crainic, A. Frangioni, and B. Gendron. Bundle-Based Relaxation Methods for Multicommodity Capacitated Fixed Charge Network Design. Discrete Applied Mathematics, 112(1-3):73-99, 2001.

[14] J. Current, M. Daskin, and D. Schilling. Discrete Network Location Models. In: Facility Location Theory: Applications and Methods. Z. Drezner and H. Hamacher Eds. Springer-Verlag., Berlin, 2001.

[15] Z. Drezner and G. O. Wesolowsky. Network Design: Selection and Design of Links and Facility Location. Transportation Research -A, 37(3):241-256, 2003.

[16] S.-S. Fan and E. Zahara. A hybrid simplex search and particle swarm optimization for unconstrained optimization. European Journal of Operational Research, 181(2):527$548,2007$.

[17] Z. Gao, J. Wu, and H. Sun. Solution Algorithm for the Bi-level Discrete Network Design Problem. Transportation Research Part B: Methodological, 39(6):479-495, 2005.

[18] R. García and D. Verastegui. Column generation procedures for the demand adjustment problem. European Journal of Operational Research, 184:860-878, 2008.

[19] R. García and D. Verastegui. Adjustment of the link travel-time functions in traffic equilibrium assignment models. Transportmetrica, DOI:10.1080/18128602.2012.669415, 2012.

[20] K. Holmberg and D Yuan. A Lagrangian Heuristic Based Branch-and-Bound Approach for the Capacitated Network Design Problem. Operations Research, 48(3):461-481, 2000.

[21] H. Hu. A Particle Swarm Optimization Algorithm for Bilevel Programming Models in Urban Traffic Equilibrium Network Design. ICCTP 2009: Critical Issues in Transportation System Planning, Development, and Management Proceedings of the Ninth International Conference of Chinese Transportation Professionals, 2009. 
[22] M. K. Jha. A Geographic Information Systems Based-Model for Highway Design Optimization. PhD thesis, College Park, University of Maryland, 2000.

[23] M. K. Jha. Using a GIS for Automated Decision-Making in Highway Cost Analysis. Transportation Research Record, 1768:260-267, 2001.

[24] M. K. Jha. Criteria-Based Decision Support System for Selecting Highway Alignments. Journal of Transportation Engineering, 129:33-41, 2003.

[25] M. K. Jha and E. Kim. Demand Considerations in Locating Transportation Facilities: A Case of Highway Alignments. Advances in Transport, 16:131-140, 2004.

[26] M. K. Jha, C. McCall, and P. Schonfeld. Using GIS, Genetic Algorithms, and Visualization in Highway Development. Computer-Aided Civil and Infrastructure Engineering, 16(6):399-414, 2001.

[27] M. K. Jha and P. Schonfeld. Geographic Information System-Based Analysis of Rightof-Way Cost for Highway Optimization. Transportation Research Record, 1719:241249, 2000.

[28] M. K. Jha and P. Schonfeld. Integrating Genetic Algorithsms and Geographic Information System to Optimize Highway Alignments. Transportation Research Record, 1719:233-240, 2000.

[29] M. K. Jha and P. Schonfeld. Trade-Offs Between Initial and Maintenance Costs of Highways in Cross-Slopes. Journal of Infrastructure Systems, 9(1):16-25, 2003.

[30] M. K. Jha and P. Schonfeld. A Highway Alignment Optimization Model Using Geographic Information Systems. Transportation Research Part A, 38:455-481, 2004.

[31] M. K. Jha, P. Schonfeld, J.-C. Jong, and E. Kim. Intelligent Road Design. WIT Press, ISBN:1-84564-003-9, SouthHampton, UK, 2006.

[32] J. C. Jong. Optimizing Highway Alignments with Genetic Algorithms. PhD thesis, College Park, University of Maryland, 1998.

[33] J. C. Jong, M. K. Jha, D. J. Lovell, and P. Schonfeld. Preliminary Highway Design with Genetic Algorithms and Geographic Information Systems. Computer Aided Civil and Infrastructure Engineering, 15:231-271, 2000.

[34] J. C. Jong and P. Schonfeld. An Evolutionary Model for Simultaneously Optimizing Three-Dimensional Highway Alignments. Transportation Research Part B, 37(2):107$128,2003$.

[35] M. W. Kang. An Alignment Optimization Model for A Simple Highway Network. PhD thesis, College Park, University of Maryland, 2008. 
[36] M. W. Kang, P. Schonfeld, and J.-C. Jong. Highway Alignment Optimization Through Feasible Gates. Journal of Advanced Transportation, 41(2):115-144, 2007.

[37] M. W. Kang, P. Schonfeld, and N. Yang. Computer-Aided Civil and Infrastructure Engineering. Journal of Advanced Transportation, 24(2):109-119, 2009.

[38] M. W. Kang, N. Yang, P. Schonfeld, and M. Jha. Bilevel Highway Route Optimization. Transportation Research Record, 2197:107-117, 2010.

[39] J. Kennedy and R. Eberhart. Particle Swarm Optimization. IEEE International Conference on Neural Networks - Conference Proceedings, 4:1942-1948, 1995.

[40] B. J. Kim, W. Kim, and B. H. Song. Improving the Computational Efficiency of Highway Alignment Optimization Models Trough a Stepwise Genetic Algorithms Approach. Transportation Research Part B, 39:339-360, 2005.

[41] E. Kim, M. K. Jha, D. J. Lovell, and P. Schonfeld. Intersection Modeling for Highway Alignment Optimization. Computer-Aided Civil and Infrastructure Engineering, 19(2):119-129, 2004.

[42] E. Kim, M. K. Jha, and P. Schonfeld. Intersection Construction Cost Functions for Alignment Optimization. Journal of Transportation Engineering, 130(2):194-203, 2004 .

[43] A. C. Lim. Transportation network design problems: An MPEC approach. PhD thesis, The Johns Hopkins University, Baltimore, Maryland, 2002.

[44] T. L. Magnanti and R. T. Wong. Network Design and Transportation Planning: Models and Algorithms. Transportation Science, 18(1):1-55, 1984.

[45] A. Marín and R. García-Ródenas. Location of Infrastructure in Urban Railway Networks. Computers $\&$ Operations Research, 36:1461-1477, 2009.

[46] MathWorks. Using Matlab Graphics. Matlab Programming. Matlab Desktop Tools and Development Environment. Version \%. MathWorks Inc, 2004.

[47] M. Minoux. Networks Synthesis and Optimum Network Design Problems: Models Solution Methods and Applications. Networks, 19(3):313-360, 1989.

[48] J. Ortúzar, and L.G. Willumsen, Modelling Transport, John Wiley \& Sons, 1994

[49] PSC. Particle Swarm Central. http://www.particleswarm.info.

[50] M. Patriksson and T. Rockafellar. A Bilevel Optimization Model and Descent Algorithm for Traffic Management. Transportation Science, 36:271-291, 2002. 
[51] G. K. D. Saharidis and M. G. Ierapetritou. Resolution method for mixed integer bi-level linear problem based on decomposition technique. Journal of Global Optimization, 44(1):29-51, 2009.

[52] R. S. Solanki, J. K. Gorti, and F. Southworth. Using Decomposition in LargeScale Highway Network Design with a Quasi-optimization Heuristic. Transportation Research Part B: Methodological, 33(12):127-140, 1998.

[53] TRB. Highway Capacity Manual. National Research Council, Washington, D.C., 2000.

[54] M. Xu, Y. Jin, and G. Ziyou. Particle Swarm Optimization Algorithm in Transport Continuous Network Design Problems. Third International Joint Conference on Computational Science and Optimization (CSO), 2010.

[55] H. Yang and M. G. H. Bell. Models algorithms for road network design: a review and some new developments. Transportation Reviews, 18(3):257-278, 1998.

[56] H. Yang, Q. Meng, and Bell M. G. H. Simultaneous estimation of the origindestination matrices and travel-cost coefficient for congested networks in a stochastic user equilibrium. Transportation Science, 35:107-123, 2001. 